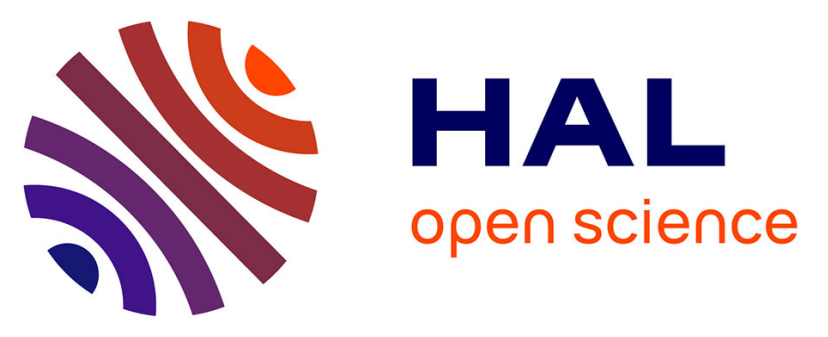

\title{
Neuroprotective effects of PACAP against paraquat-induced oxidative stress in the Drosophila central nervous system
}

Khadija Hajji, Ali Mteyrek, Jun Sun, Marlène Cassar, Sana Mezghani, Jérôme Leprince, David Vaudry, Olfa Masmoudi-Kouki, Serge Birman

\section{To cite this version:}

Khadija Hajji, Ali Mteyrek, Jun Sun, Marlène Cassar, Sana Mezghani, et al.. Neuroprotective effects of PACAP against paraquat-induced oxidative stress in the Drosophila central nervous system. Human Molecular Genetics, 2019, 28, pp.1905 - 1918. 10.1093/hmg/ddz031 . hal-02396061

\section{HAL Id: hal-02396061 https://hal.science/hal-02396061}

Submitted on 10 Dec 2019

HAL is a multi-disciplinary open access archive for the deposit and dissemination of scientific research documents, whether they are published or not. The documents may come from teaching and research institutions in France or abroad, or from public or private research centers.
L'archive ouverte pluridisciplinaire HAL, est destinée au dépôt et à la diffusion de documents scientifiques de niveau recherche, publiés ou non, émanant des établissements d'enseignement et de recherche français ou étrangers, des laboratoires publics ou privés. 


\section{oxidative stress in the Drosophila central nervous system}

4 Khadija Hajji $^{1,2}$, Ali Mteyrek ${ }^{1}$, Jun $\operatorname{Sun}^{1}$, Marlène Cassar ${ }^{1}$, Sana Mezghani², Jérôme

5 Leprince ${ }^{3,4}$, David Vaudry ${ }^{3,4}$, Olfa Masmoudi-Kouki ${ }^{2,}{ }^{*}$ and Serge Birman ${ }^{1, *}$

$6{ }^{1}$ Genes Circuits Rhythms and Neuropathology (GCRN) Group, Brain Plasticity Unit, CNRS,

7 ESPCI Paris, Labex Memolife, PSL Research University, 10 rue Vauquelin, 75005 Paris, France

8 2University Tunis E1 Manar, Faculty of Sciences of Tunis, LR18ES03, Laboratory of

9 Neurophysiology, Cellular Physiopathology and Biomolecules Valorisation, 2092 Tunis, Tunisie

$10{ }^{3}$ Laboratory of Neuronal and Neuroendocrine Communication and Differentiation, INSERM

11 U1239, Institute for Research and Innovation in Biomedicine (IRIB), Normandy University,

1276821 Mont-Saint-Aignan, France

$13{ }^{4}$ Regional Cell Imaging Platform of Normandy (PRIMACEN), Normandy University,

14 UNIROUEN, INSERM, 76821 Mont-Saint-Aignan, France

17 *Address for correspondence and reprint requests to Serge Birman (serge.birman@espci.fr) and

18 Olfa Masmoudi-Kouki, (olfa.masmoudi@fst.utm.tn) 


\section{Abstract}

25 Parkinson's disease (PD) is a progressive neurodegenerative movement disorder that can arise

26 after long-term exposure to environmental oxidative stressors, such as the herbicide paraquat

27 (PQ). Here we investigated the potential neuroprotective action of vertebrate pituitary adenylate

28 cyclase-activating polypeptide (PACAP) against PQ in Drosophila. We found that pretreatment

29 with this neuropeptide applied to the ventral nerve cord (VNC) at low doses markedly extended

30 the survival of wild-type decapitated flies exposed to neurotoxic levels of PQ or dopamine (DA).

31 In contrast and interestingly, application of a PACAP receptor antagonist, PACAP-6-38, had

32 opposite effects, significantly decreasing the resistance of flies to PQ. PACAP also reduced PQ-

33 induced caspase activation and reactive oxygen species (ROS) accumulation in the VNC. We

34 then searched for the endogenous neuropeptide receptor potentially involved in PACAP-mediated

35 neuroprotection in Drosophila. Knocking down the gene encoding the receptor Han/PDFR of the

36 neuropeptide pigment-dispersing factor (PDF) in all neurons conferred to flies higher resistance

37 to PQ, whereas PDFR downregulation restricted to PDF or DA neurons did not increase PQ

38 resistance, but remarkably suppressed the neuroprotective action of PACAP. Further experiments

39 performed with $P d f$ and $P d f r$-deficient mutant strains confirmed that PDF and its receptor are

40 required for PACAP-mediated neuroprotection in flies. We also provide evidence using split-

41 GFP reconstitution that PDF neurons make synaptic contacts onto DA neurons in the abdominal

42 VNC. Our results, therefore, suggest that the protective action of PACAP against PQ-induced

43 defects in the Drosophila nervous system involves the modulation of PDFR signaling in a small

44 number of interconnected neurons. 


\section{Introduction}

48 Parkinson's disease (PD) is a progressive neurodegenerative movement disorder believed to

49 develop as a result of an interplay between genetic and environmental factors (1). The

50 identification of genetic factors that modulate an individual's sensitivity to environmental agents

51 can lead to better risk assessment and understanding of the disease mechanisms $(2,3)$. The motor

52 symptoms of PD are primarily caused by the degeneration of dopaminergic (DA) neurons

53 innervating the striatum whose cell bodies are located in the midbrain substantia nigra pars

54 compacta (SNpc) (4). Epidemiological studies have suggested that long-term exposure to

55 pesticides such as the herbicide paraquat (1,1'-dimethyl-4-4'-bipyridinium, PQ) is associated with

56 an increased risk of developing PD (5-7). In accordance with this, PQ has been shown to induce

57 the degeneration of nigrostriatal DA neurons in rodents (8-12), due to increased production of

58 superoxide ions and oxidative damage (13-19).

59 The neuropeptide pituitary adenylate cyclase-activating polypeptide (PACAP), originally

60 identified as a highly potent adenylate cyclase-stimulating peptide with two biologically active

61 isoforms, PACAP-38 and PACAP-27, exerts a wide range of actions via the activation of three G

62 protein-coupled receptors (GPCRs): PAC1-R, which is PACAP-specific, and VPAC1-R and

63 VPAC2-R indifferently activated by PACAP or vasoactive intestinal polypeptide (VIP) (20).

64 PACAP is known as a powerful anti-apoptotic, anti-inflammatory and anti-oxidative agent (20-

65 22). By reducing apoptosis, PACAP exhibits neuroprotective effects in diverse cellular and

66 animal models of neuronal damage, including cerebral ischemia, brain injury, Alzheimer's

67 disease and PD (23-27). In particular, it has been reported that PQ-induced loss of DA neurons

68 was more severe in the SNpc of PACAP knock-out mice compared to wild-type animals,

69 associated with increased microglial activation (25). This result indicates that endogenous 
70 PACAP normally acts to maintain the integrity of SNpc DA neurons, an action that may be in

71 part linked to its ability to regulate microglia and immune cells $(25,28)$.

72 One model system that has proven surprisingly tractable for neurodegenerative diseases is the

73 fruit fly Drosophila melanogaster, which is now widely used to search for novel therapeutic

74 targets and neuroprotective compounds (29-32). Previous reports have demonstrated that

75 Drosophila exposed to environmental pro-oxidant toxins, such as rotenone or PQ, reproduce PD

76 features, including selective degeneration of DA neurons (33-40). Neuropeptides are abundant in

77 the Drosophila brain where they play essential roles in various physiological functions and

78 behavior neuromodulation $(41,42)$. The fly amnesiac (amn) gene potentially encodes three

79 neuropeptides, one of which displays a low (10\%) identity to PACAP-38 and was previously

80 considered as a possible PACAP orthologue (43-45), but there is still no direct evidence for the

81 presence of amn-encoded neuropeptides in Drosophila (41). Nevertheless, a PACAP-like

82 immunoreactivity was detected in the nervous system of various invertebrates $(45,46)$, and at the

83 Drosophila larval neuromuscular junction (47). Focal application of vertebrate PACAP-38 to this

84 glutamatergic synapse triggered two temporally distinct responses: an immediate depolarization

85 and a large enhancement of potassium current (47). PACAP-38 also enhanced L-type calcium-

86 current in larval muscles via the adenylate cyclase-cAMP-protein kinase A (PKA) transduction

87 pathway (48). No specific receptor has been described to date that could mediate these effects.

88 However, the receptor Han/PDFR of the neuropeptide pigment-dispersing factor (PDF), is

89 homologous to the mammalian VIP/PACAP receptor VPAC2-R (49-51), and it has binding

90 affinity for PACAP (51). Interestingly, both PACAP and PDF are "circadian neuropeptides" that

91 play important roles in the regulation and entrainment of circadian rhythms in mammals and

92 insects, respectively $(42,52,53)$. Circadian clocks are highly conserved from flies to humans.

93 They control rhythms in most physiological function, with free-running periods close to $24 \mathrm{~h}$. 
94 Clock disruption is suspected to reduce lifespan and quality of life during aging, and

95 neurodegenerative disorders, like PD, often disrupt biological clocks early on $(54,55)$.

96 Here we assessed the protective effect of PACAP against PQ neurotoxicity in Drosophila and

97 we searched for the G protein-coupled receptor (GPCR) mediating the action of PACAP in the

98 central nervous system (CNS). We show evidence that the neuroprotective action of PACAP is

99 conserved in Drosophila and specifically involves PDFR signaling in PDF and DA neurons.

\section{Results}

\section{PACAP protects wild-type flies from PQ-induced neurotoxicity}

103 To test the potential neuroprotective action of PACAP against PQ, administration via diet was

104 not feasible because neuropeptides are vulnerable to intestinal peptidases. Therefore, we applied

105 the neuropeptide onto the exposed ventral nerve cord (VNC) of decapitated flies, which can

106 maintain a normal standing posture and survive up to 3 days when kept in a humid environment

107 at $25^{\circ} \mathrm{C}(29,39,56,57)$. We have previously shown in this preparation that a 5-s application of a

108 drop of $80 \mathrm{mM}$ PQ diluted in Ringer's solution onto the exposed VNC killed about 30-40\% of

109 the flies in $2 \mathrm{~h}(39)$.

110 Consistent with our previous report, PQ application decreased significantly the survival of

111 decapitated flies and this effect was observed from the first $30 \mathrm{~min}$ after exposure (Fig. 1A).

112 Remarkably, a 5-s application of a drop of $2 \mu \mathrm{M}$ PACAP (P) diluted in Ringer's solution, but not

113 of Ringer's only (R), 30 min before that of PQ, markedly protected flies against PQ-induced

114 lethality (Fig. 1A). The protective action of PACAP remained significant until 3 hours after PQ

115 exposure. A maximal effect was observed at $120 \mathrm{~min}(+30.3 \%, p<0.001)$ for the survival of $\mathrm{P}+$

116 PQ $v s \mathrm{R}+\mathrm{PQ}$ flies. Note that PACAP had no effect by itself on fly survival $(\mathrm{P}+\mathrm{R} v s \mathrm{R}+\mathrm{R}$, Fig. 
117 1A). We have previously reported that DA itself can also be lethal when applied at $20 \mathrm{mM}$ or 118 higher to VNC in the same way as PQ (39). Here we observed that PACAP pretreatment fully 119 rescued the flies from the lethality induced by the application of a drop of DA at $35 \mathrm{mM}$ to the 120 VNC (Supplementary Material, Fig. S1). These results show that PACAP is protective against 121 both PQ- and DA-induced neurotoxicity in the Drosophila CNS.

122 The N-terminal residues of PACAP (PACAP-38 and PACAP-27) are essential for its

123 biological activity. Consequently, the deleted peptide PACAP-6-38 is inactive and it is also a 124 potent antagonist of the PACAP-specific receptor PAC1-R (58). We therefore investigated the 125 effect of PACAP-6-38 on PQ sensitivity in Drosophila. Strikingly, we found that decapitated 126 flies pre-treated for 30 min with $2 \mu \mathrm{M}$ PACAP-6-38 were significantly more susceptible to PQ 127 than control flies (Supplementary Material, Fig. S2). In the presence of PACAP-6-38, fly 128 survival was reduced by $20 \%$ and $19 \%(\mathrm{p}<0.001) 60$ and 90 min after PQ application, 129 respectively. This shows that PACAP and PACAP6-38 applied to the VNC have opposite effects 130 on oxidative stress resistance in Drosophila.

131 To learn more about the mechanisms of PACAP-mediated protection, we analyzed the effects

132 of this neuropeptide on PQ-induced increase in reactive oxygen species (ROS) and caspase 133 activation levels in the VNC. We estimated ROS levels by dihydroethidium (DHE) staining in 134 wild-type Canton-S (CS) flies pre-treated or not with PACAP. We found that PACAP-treated 135 flies exhibited significantly lower dye fluorescence in the VNC after 90 min of PQ exposure ($13670 \% ; p<0.01$ ) (Fig. 1B and C). Similarly, using the CaspaseTracker biosensor system (59), which 137 detects activation of the effector caspases, such as DrICE, that cleave protein substrates at a 138 DQVD motif, we observed that PACAP pre-treatment significantly decreased the number of 139 activated caspase-positive VNC cells in PQ-exposed decapitated flies $(-40 \% ; p<0.01)$ (Fig. 1D 140 and E). Therefore, PACAP efficiently reduced the level of oxidative stress and caspase activation 
141 under acute PQ intoxication in the Drosophila CNS.

\section{Neuronal downregulation of the PDF receptor decreases PQ susceptibility}

143 In order to search for the neuropeptide receptor potentially involved in PACAP-mediated

144 neuroprotection, genes encoding G protein-coupled receptors for several neuropeptides of the

145 Drosophila CNS were downregulated by targeted RNA interference (RNAi) with the pan-

146 neuronal driver elav-Gal4 and co-expression of the RNAi booster Dcr-2, and adult fly survival

147 was monitored 90 min after application of PQ (Table 1). Downregulation of Dh31-R, Dh44-R1,

$148 D h 44-R 2, F M R F a R$, and $s N P F-R$ did not affect fly PQ susceptibility, or the protective effect of

149 PACAP from PQ-provoked neurotoxicity. In contrast, silencing the gene han/Pdfr, which

150 encodes the receptor for the neuropeptide pigment-dispersing factor (PDF), significantly

151 increased Drosophila resistance to PQ, and PACAP pre-treatment did not further increase the

152 survival rate of these flies (Table 1). We then analyzed the survival kinetics of flies in which Pdfr

153 was either downregulated (Fig. 2A) or overexpressed (Fig. 2B) in all neurons, respectively. This

154 confirmed that Pdfr silencing by RNAi significantly counteracted PQ-induced lethality from the

155 first hour of exposure, while there was no detectable effect of PACAP in these conditions (Fig.

156 2A). In contrast, $P d f r$ overexpression in all neurons did not cause a noticeable effect on PQ

157 susceptibility (Fig. 2B).

158 The receptor PDFR is widely expressed in the Drosophila clock neurons, and it is also

159 expressed in non-clock brain cells as well as in peripheral clock cells $(60,61)$. Knocking down

$160 P d f r$ by RNAi with the clock cell driver tim-Gal4 $(62,63)$ conferred to decapitated flies a higher

161 resistance to PQ, an effect that was highly significant between 90 and 150 min after PQ

162 application (Fig. 2C). Such a protection ( $t i m>P d f r^{\mathrm{RNAi}}, D c r-2$ flies, R + PQ) was comparable to

163 that induced by PACAP pre-treatment on the controls $\left(P d f r^{\mathrm{RNAi}} /+, D c r-2 /+, \mathrm{P}+\mathrm{PQ}\right)$. Combining 
164 the effects of PACAP pre-treatment and Pdfr knock down (i.e. $t i m>P d f r^{\mathrm{RNAi}}, D c r-2, \mathrm{P}+\mathrm{PQ}$ ) did

165 not further increase neuroprotection, indicating that these effects are apparently not additive (Fig.

166 2C). Note that a similar level of protection against PQ was observed when Pdfr RNAi was

167 expressed with elav-Gal4 or tim-Gal4 in a parallel experiment (Fig. 2D). We also tested the effect 168 of silencing the PDF receptor in glial cells using repo-Gal4, but the survival rate of these flies

169 was not increased, suggesting that this cell type is not involved in the protective effect

170 (Supplementary Material, Fig. S3).

171 It may seem surprising that knocking down Pdfr with the clock cell driver tim-Gal4 had a

172 protective effect on decapitated flies, because no clock neurons are present in the adult

173 Drosophila VNC (63). However, it has been reported that tim-GAL4 drives GFP expression in

174 some neurons that do not express the TIM protein at detectable levels in the adult brain $(63,64)$.

175 We have checked that this is this also the case in the adult VNC. The pattern of membrane-

176 associated GFP in the CNS of $\operatorname{tim}>m C D 8:: G F P, n S y b:: G F P$ flies revealed that tim-Gal4

177 expresses in several subsets of neurons in the abdominal ganglia (Supplementary Material, Fig.

178 S4A). This region of the VNC also contain the abdominal (Abd) PDF neurons (65) and subsets of

179 DA neurons (39). PDF co-immunostaining showed that tim-Gal4 apparently does not label the

180 Abd PDF neurons (Supplementary Material, Fig. S4B), in agreement with the observation that

181 these cells do not express a molecular clock (65).

182

183 Neuroprotection by PACAP requires PDF and the PDF receptor

184 We then tested the PQ resistance of a $P d f r$ null mutant strain, $P d f r^{5304}$ (49). This strain

185 appeared slightly more resistant than wild-type CS controls between 30 and 90 min after PQ

186 application, but the effect was not statistically significant (Fig. 3A). Strikingly, we observed that

187 PACAP pre-treatment did not induce protection against PQ in $P d f r^{5304}$ mutant flies, suggesting 
188 that PDF receptor signaling is required for the protective action of PACAP (Fig. 3A). To further 189 assess this idea, we used the $P d f^{01}$ null mutant line that does not express PDF (66). PACAP190 mediated protection was indeed abolished in this strain (Fig. 3B). Therefore, PACAP requires 191 both PDF and PDFR expression to promote neuroprotection.

192 We noted that the lack of PDF or PDFR did not increase PQ resistance (Fig. 3B), in contrast to 193 the effect induced by RNAi-mediated $P d f r$ knock down in all neurons or clock cells, suggesting 194 that $P d f r$ downregulation in neuronal subsets can induce different effects than its total absence. 195 This result may suggest that it is a constitutive, and not PDF-stimulated, activity of PDFR that 196 increases PQ susceptibility in wild-type flies. Alternatively, spontaneous mutations accumulated 197 over generations (67) might have compensated for the lack of PDFR signaling for this specific 198 phenotype in the $P d f f^{5304}$ and $P d f^{01}$ mutants. To test these possibilities, we silenced PDF neuron 199 activity by expressing the hyperpolarizing potassium channel Kir2.1 with the Pdf-Gal4 driver 200 (68). Interestingly, this led to significantly increased PQ resistance between 30 and 90 min of 201 exposure (highest at 90 min: $+27.7 \%, p<0.001$ ) (Fig. 3C), indicating that PDF neuron activity is 202 at least in part involved in PDFR-induced increase in PQ susceptibility. The rescue was not as 203 strong as in the case of the $P d f^{\text {RNAi }}$ flies, which suggests that a constitutive activity of PDFR may 204 indeed contribute to decrease oxidative stress resistance in wild-type flies. Furthermore, we 205 observed that pre-treatment with $2 \mu \mathrm{M}$ (Fig. 3D), or even $20 \mu \mathrm{M}$ (Supplementary Material, Fig. 206 S5), PDF had no effect on PQ resistance, suggesting that to increase PQ susceptibility in the 207 CNS, not only PDF but also another signaling molecule is needed. This other molecule could be 208 the unidentified classical neurotransmitter that is co-released with PDF by PDF neurons $(69,70)$.

\section{The protective action of PACAP requires PDFR expression in PDF and DA neurons}

210 PDFR is known to be expressed in the PDF-producing neurons themselves, allowing an 
211 autocrine modulation of these cells by the neuropeptide they release $(60,71)$. We observed that

212 silencing PDFR selectively in PDF neurons with Pdf-Gal4 (72) did not lead to increased PQ

213 resistance, but fully prevented PACAP neuroprotective effects (Fig. 4A). Therefore, PDFR

214 expression in the PDF neurons appears essential for PACAP-mediated neuroprotection. A quite

215 similar effect was observed when we restricted PDFR knock down to the Abd PDF neurons of

216 the VNC, using the Dot-Gal4 driver (65). PQ susceptibility did not increase while the protective

217 effect of PACAP was lost (Fig. 4B). This suggests that the presence of PDFR in the Abd PDF

218 neurons is required for PACAP-mediated neuroprotection. We next investigated whether other

219 neuronal cells could be involved in the protective effect of PACAP against PQ oxidative stress.

220 Since DA neurons are very sensitive to PQ toxicity (39), we evaluated the effect of $P d f r$

221 downregulation in these cells using TH-Gal4 (73). Interestingly, this also led to loss of PACAP

222 protective effects, without increasing PQ resistance (Fig. 4C). PDFR appears therefore required

223 both in PDF and DA neurons for PACAP-mediated neuroprotection.

224 These results prompted us to search for potential synaptic connections between the PDF and

225 DA neurons in the Drosophila VNC by using split-GFP reconstitution (74). The fusion protein n-

226 syb::spGFP1-10, which is targeted to synaptic vesicles (75), was expressed in PDF neurons with

$227 P d f$-Gal4 and the other membrane-associated GFP moiety, CD4::spGFP11, in DA neurons using

228 the $T H$-LexA driver, respectively $\left(P D F>n S y b:: G F P_{1-10}+T H>C D 4:: s p G F P_{11}\right.$ flies).

229 Reconstituted split GFP (rsGFP) fluorescence was clearly visible in prominent bilateral axonal

230 bundles located laterally in the abdominal ganglia (Fig. 5, arrowheads). In contrast, no specific

231 rsGFP signal could be detected in the abdominal ganglia of $T H>n S y b:: G F P_{1-10}+$

$232 P D F>C D 4: \because s p G F P_{11}$ flies (data not shown). These observations suggest that PDF neurons

233 synapse onto DA neurons in this region of the VNC and not the reverse. Interestingly, these

234 lateral axons appear to be also targeted by tim-Gal4 (Fig. S4, arrows), suggesting that the 
235 protective effect in the VNC of $t i m>P d f r^{\mathrm{RNAi}}$ flies could specifically result from PDFR

236 downregulation in these neurons. These lateral abdominal axons could be part of a neuronal

237 network controlling oxidative stress susceptibility in Drosophila.

\section{Discussion}

\section{The neuroprotective function of PACAP is conserved in Drosophila}

241 The neuropeptide PACAP has been widely shown to have potent antioxidant and anti-

242 apoptotic properties in several neuronal cell types in vitro, including cultured rat cerebellar

243 granule neurons and astroglial cells, and in vivo models of ischemia, stroke, traumatic brain

244 injury, multiple sclerosis, and of various neurodegenerative diseases such as Huntington's chorea,

245 Alzheimer's disease and PD $(21,22,76-80)$. Its neuroprotective action against DA neuron 246 degeneration has been demonstrated in drug-induced PD models in rodents, using pro-oxidant

247 toxins such as 1-methyl-4-phenyl-1,2,3,6-tetrahydropyridine (MPTP), 6-hydroxydopamine (6248 OHDA), rotenone and PQ $(22,81)$. PACAP injection also prolonged the survival of pond snails

249 (Lymnaea stagnalis) exposed to rotenone, and partly rescued the reduction in locomotor activity

250 and CNS DA level triggered by the toxin in this invertebrate model (82).

251 PACAP is also a survival-promoting peptide in mammalian species, playing major and pivotal

252 roles in immunity and inflammation, and extending thereby the survival of mice with ileitis

253 (intestinal inflammation) (83) and rats subjected to kidney ischemia-reperfusion (84). PACAP ${ }^{-/-}$

254 null mutant mice show a high early-mortality rate, in part related to increased susceptibility to

255 cold stress (85), and are poorly resistant to doxorubicin-induced myocardial damage (86).

256 Moreover, $\mathrm{PACAP}^{-/}$mice are more sensitive to $\mathrm{PQ}$-induced DA neuron depletion, suggesting

257 that endogenous PACAP has a physiological neuroprotective action in the brain (25). 
Here we examined the effect of PACAP in a widely used sporadic PD model induced by PQ

259 exposure in Drosophila $(34,37,39,87)$. Since the fly orthologue of PACAP has not yet been

260 identified with confidence (41), we used the amino acid sequence of PACAP-38, which is

261 identical in all mammals, and because PACAP could not cross the intestinal barrier and remain

262 intact, we directly applied the neuropeptide to the exposed VNC of decapitated Drosophila

263 before PQ exposure, using a previously established procedure (39). We repeatedly observed that

264 PACAP pre-treatment at $2 \mu \mathrm{M}$ prolonged the survival of wild-type decapitated Drosophila

265 exposed to PQ and that it was also protective against neurotoxic DA concentrations. In contrast,

266 an opposite effect was observed, that is, an increase in fly susceptibility to PQ, when the

267 antagonist PACAP-6-38 was applied, which is structural similar to PACAP-38 except for a short

268 deletion of 5 amino acids in the N-terminal that suppresses its activity. This indicates that the

269 neuroprotection conferred by PACAP in flies is a genuine and specific effect that requires the

270 same structural domain than for its protective action in vertebrate systems. In addition, the

271 opposite effect of the PACAP antagonist may suggest that a still unidentified, most likely

272 peptidic, endogenous molecule, binds on the same receptor as PACAP and contributes to

273 oxidative stress resistance in the Drosophila nervous system. We attempted to overexpress

274 amnesiac in neurons (elav $>a m n$ flies), but this had no effect on PQ resistance (data not shown),

275 suggesting that amn is not the functional counterpart of PACAP in the flies.

276 As was shown in mammalian cultured cerebral cells, PACAP is able: $i$ ) to stimulate

277 endogenous antioxidant system by increasing the level of glutathione, the major free radical

278 scavenger in the brain, and superoxide dismutase (SOD) and catalase activity, and ii) to prevent

279 inhibition of endogenous ROS defense system under oxidative stress $(77,79,80)$. This suggests

280 that the neuropeptide could block ROS overproduction and dampen oxidative stress through an

281 upregulation of ROS-detoxifying enzymes in vivo. Consistent with this observation, it has been 
282 shown that enhancing antioxidant SOD2 and glutathione peroxidase 1 (GPX1) activity by

283 PACAP provided protection against oxidative damage and neuroprotection in mice with

284 traumatic brain injury (88) and that inhibition of the endogenous antioxidant system in astrocytes

285 suppresses the cell survival-promoting effect of PACAP under oxidative conditions (80). Here

286 we show accordingly that PACAP efficiently alleviated the increase in ROS accumulation

287 triggered by PQ in the fly VNC. A possible effect of PACAP on the activity of endogenous

288 antioxidant systems in PQ-treated Drosophila could thus be a key player in its mechanisms of

289 action. These results are striking because PQ is a very strong inducer of ROS production in

290 Drosophila $(18,39,40,87,89)$, and we found here that a low dose of PACAP was sufficient to

291 largely prevent the neurotoxic effects of this herbicide.

292 Previous work demonstrated that PACAP prevents cerebellar granule neurons from apoptotic

293 cell death through inhibition of caspase-3 activity (90). Comparably, in the mollusk Helix

294 pomatia, PACAP efficiently attenuated caspase-3 activation induced by cytotoxic levels of DA

295 (91). We have thus investigated the effect of PACAP on PQ-induced activation of effector

296 caspases in the Drosophila CNS with the CaspaseTracker biosensor system (59). PQ is known to

297 cause an increase in caspase activity, both in mice and Drosophila $(40,92,93)$. Pre-treatment of

298 the flies with the neuropeptide PACAP significantly decreased caspase activation under PQ in the

299 VNC, possibly resulting from oxidative stress reduction. It has been reported indeed that PACAP

300 can regulate the expression of Bcl-2 family members to prevent apoptotic cell death provoked by

301 oxidative insult in neuronal cells $(21,93,94)$.

302 PDFR signaling significantly contributes to PQ neurotoxicity in the Drosophila CNS

303 How can such a tiny amount of PACAP (40,000 times less than PQ) prevent oxidative stress-

304 induced defects in the whole VNC and thus significantly prolong the survival of PQ-intoxicated 
305 decapitated Drosophila? To explore the mechanisms of action of this neuropeptide, we first used

306 pan-neuronal RNAi downregulation to search among GPCR receptors of known fly

307 neuropeptides for one that might be implicated in the protective effect of PACAP. Among the six

308 GPCRs that were tested, only PDFR was identified as being involved in the modulation of PQ

309 sensitivity and PACAP-mediated neuroprotection. Indeed, downregulating this receptor in all

310 neurons with elav-Gal4, or in tim-Gal4-targeted cells, was sufficient to protect efficiently the

311 flies from PQ-induced early lethality. This indicates that PDFR signaling, which acts through

312 elevated cAMP/PKA activity $(51,69,71,95-99)$ and modulation of $\mathrm{Ca}^{2+}$ level and activities (51,

$31396,98,100,101)$, potently contributes to PQ susceptibility in Drosophila. In contrast, Pdf ${ }^{3304}$

314 and $P d f^{01}$ null mutants did not appear more resistant to PQ than wild-type flies, which might be

315 related to compensating spontaneous mutations accumulated in the mutant genomes over

316 generations (67), or, alternatively, to the absence of PDFR signaling at all stages and in all cells

317 in the mutants compared to a relative downregulation in neurons only in the RNAi experiment.

318 Note that $P d f r$ knock down by RNAi in PDF neurons was not sufficient to decrease PQ

319 susceptibility, indicating that enhanced PQ resistance requires targeting other $P d f r$-expressing

320 neurons.

321 The fact that PDF release is involved in PDFR-induced increase in PQ susceptibility was

322 suggested by the Kir2.1 experiment, in which in vivo silencing of PDF neurons with this

323 potassium channel significantly enhanced PQ resistance of the flies. However, we show here that

324 direct PDF application to the VNC of decapitated flies had no effect on PQ susceptibility, and we

325 also observed that pan-neuronal expression of membrane-tethered PDF (t-PDF) (99) similarly did

326 not affect PQ sensitivity of the flies (data not shown). This suggests that an additional molecule is

327 required, potentially the classical transmitter that is co-released with PDF by PDF neurons (69).

328 Indeed, these last authors showed the release of this co-transmitter is also increased by PDFR 
auto-receptor activation.

330 By which mechanisms could PDFR signaling contribute prominently to PQ neurotoxicity is a

331 matter of speculation. It has been demonstrated that PDF can convey signals that lead to

332 activation of caspases and enhanced neurodegeneration in target cells in several parts of the

333 Drosophila brain (102). This occurred in young flies with circadian dysfunction and in older

334 wild-type flies. These effects correlated with enhanced tauopathy in a fly model of Alzheimer's

335 disease (102). We have also recently reported that a mutation in the circadian gene $C l o c k\left(C l k^{\mathrm{AR}}\right)$,

336 or RNAi-induced Clk knock down in PDF neurons, led to accelerated age-related locomotor

337 decline and apoptosis-related loss of DA neurons in the PPL1 cluster, all effects which were

338 found to be strictly dependent on PDFR signaling (103). These and other findings suggest the

339 implication of the circadian system and PDF/PDFR signaling in age-related loss of physical or

340 cognitive abilities and enhanced neurodegeneration in Drosophila (104-106).

341 The ROS-related disturbance induced by PQ exposure could trigger the depolarization of PDF

342 neurons, thus promoting caspase activation and apoptosis in Pdfr-expressing target cells, and the

343 increased resistance to PQ when $P d f r$ is downregulated in tim-expressing neurons might be the

344 result of the negative regulation of these molecular events. PDF neuron overactivation could

345 directly or indirectly lead to similar defects in DA neurons, which would result in abnormally

346 high level of DA release, inducing cytotoxic and excitotoxic effects through overstimulation of

347 the $\mathrm{D}_{1 / 5}$-like DA receptor DAMB, which, as we have previously reported, can provoke calcium

348 release in the cytosol of glutamatergic neurons through the ryanodine receptor and finally

349 contribute to nervous system failure in PQ-intoxicated flies (39).

350 PACAP neuroprotection depends on PDFR signaling in PDF and DA neurons

351 We observed that PACAP pre-treatment did not prolong the survival of PQ-exposed null Pdf 
352 or $P d f f^{5304}$ mutants that are deficient for PDF and PDFR, respectively, at variance with the potent

353 protective effect of this neuropeptide on wild-type flies. No effect of PACAP could be detected

354 after $P d f r$ downregulation in all neurons or in all clock cells, but this could be because PQ

355 resistance was already much increased in these flies. In contrast, targeting Pdfr RNAi in PDF

356 neurons selectively did not increase PQ resistance and instead fully prevented PACAP protective

357 effects. Furthermore, restricting Pdfr RNAi knock down in the VNC Abd PDF neurons also

358 suppressed the beneficial effect of PACAP pre-treatment for fly survival under acute oxidative

359 stress. Although PDFR has binding affinity for PACAP in vitro (51), PACAP is apparently not an

360 agonist of this receptor since it has been shown that its application to dissected brain did not

361 significantly alter the FRET signal of a cAMP sensor in the PDF neurons that express PDFR 362 (71).

363 In adult Drosophila, there are only 24 PDF-expressing neurons in the CNS: four or five large

$364\left(1-\mathrm{LN}_{\mathrm{v}} \mathrm{s}\right)$ and four small $\left(\mathrm{s}-\mathrm{LN}_{\mathrm{v}} \mathrm{s}\right)$ ventral lateral neurons per hemisphere in the brain, and four

365 large (1-Abd) and four small (s-Abd) abdominal neurons in the $\mathrm{VNC}(98,107,108)$. It is known

366 that brain PDF neurons (the s-LN $\mathrm{LN}_{v}$ and some of the 1-LN $\mathrm{LN}_{\mathrm{v}}$ ) express PDFR autoreceptors (60,

367 69). The l-Abd and s-Abd PDF neurons do not express the molecular clock and the PDF

368 neuropeptides released from these neurons are not required for locomotor rhythms (65).

369 Nevertheless, both the brain $\mathrm{LN}_{\mathrm{v}} \mathrm{s}$ and VNC Abd PDF neurons contribute to the normal phasing

370 of the molecular clock in the oenocytes, which are pheromone-producing peripheral clock cells

$371(61,98)$. Furthermore, only the PDF released from the Abd PDF neurons is necessary to

372 maintain wild-type expression levels of male sex pheromones (61).

373 Since we have performed our experiments on decapitated flies, it is natural to consider that the

374 PDF neurons in which PDFR is required for PACAP neuroprotection are specifically the Abd

375 neurons. This seems to be supported indeed by the fact that $\operatorname{Dot}>P d f r^{\mathrm{RNAi}}$ decapitated flies are not 
376 sensitive to PACAP. However, we cannot exclude that long-term PDFR deficiency in the brain

377 PDF neurons might have a distant influence on other organs, including the VNC, that would

378 contribute to PACAP-induced protection. Such an influence would have to be indirect because it

379 is known that the brain PDF neurons do not send descending projections to the VNC (108). It is

380 quite possible that, like for the phasing of oenocyte molecular clock, full PACAP neuroprotection

381 requires PDFR expression in both the brain and VNC PDF neurons. Note that the same issue

382 arises for the higher PQ resistance of decapitated flies in which Pdfr was downregulated with tim-

383 Gal4. It is possible that the higher PQ resistance of $t i m>P d f r^{\mathrm{RNAi}}$ flies results from long-term

384 PDFR deficiency in the brain clock neurons that would make decapitated flies less susceptible to

385 PQ neurotoxicity by a systemic mechanism. However, we show here that tim-Gal4 expresses in

386 subsets of neurons in the abdominal ganglia, some of which could express PDFR (the PDFR

387 pattern has not been described in the VNC to date) and be directly involved in the control of PQ

388 susceptibility in the CNS.

389 Finally, we observed that downregulating Pdfr in DA neurons also prevented PACAP-

390 mediated neuroprotection. As in the case of PDF neurons, both the brain and VNC DA neurons

391 could be involved in this effect, even though we used decapitated flies for the survival test. A

392 recent report provided evidence that a subset of dopaminergic neurons respond to PDF and that

393 the s-LN $\mathrm{V}_{\mathrm{v}}$ axons and some brain DA neurons form synaptic contacts in the brain (109). As

394 mentioned above, we have also previously shown that $C l k$ downregulation in the sLNvs leads to

395 PDFR-dependent degeneration of DA neurons (103). Here we show using split-GFP

396 reconstitution that Abd PDF neurons also contact DA neurons in the abdominal region of the

397 VNC, before these PDF neurons send projections out of the VNC (98). The synapses appear

398 distributed along large bilateral axonal bundles. The fact that the silencing of PDFR in either PDF

399 or DA neurons has no effect on the sensitivity of the flies to PQ, may suggest that PDFR has to 
400 be knocked down in both cell types and probably in other clock neurons to increase PQ

401 resistance. Remarkably, downregulating PDFR either in PDF or DA neurons was sufficient to

402 fully prevent PACAP neuroprotection, indicating that PACAP must act on these two neuronal

403 subsets to protect flies from PQ.

404 In conclusion, our current findings indicate that the neuropeptide PACAP offers a potent

405 protection against PQ neurotoxicity in Drosophila and that this effect can be ascribed to the

406 modulation of PDF/PDFR signaling in specific neuronal subsets. Further studies are required to

407 characterize the signaling pathways that trigger this neuroprotective effect and so better

408 understand the neuronal circuits and mechanisms controlling oxidative stress susceptibility in the 409 Drosophila CNS.

410

411 Materials and methods

412 Drosophila culture and strains

413 Fly stocks were raised at $25^{\circ} \mathrm{C}$ on standard cornmeal-yeast-agar medium supplemented with

414 methyl-4-hydroxy-benzoate as a mold protector, under $12 \mathrm{~h}-12 \mathrm{~h}$ light-dark cycle and $70 \%$

415 humidity. The following strains were used: Canton-S (CS) as wild type, $P d f f^{5304}(49), P d f^{01}(66)$,

416 elav-GAL4 (110), repo-GAL4 (111), TH-Gal4 (73), TH-LexA (112) (kindly provided by Dr.

417 Ronald L. Davis), tim-Gal4 (62), Pdf-Gal4 (72), Dot-Gal4 (65), UAS-Pdfr (49) (kindly provided

418 by Dr. François Rouyer), UAS-Kir2.1 (113) (kindly provided by Dr. Sean Sweeney), recombined

419 UAS-mCD8::GFP, UAS-nSyb::GFP (114), and from the Bloomington Drosophila Stock Center

420 (BDSC): elav-Gal4; UAS-Dcr-2 (BDSC \#25750); UAS-Dcr-2; Pin ${ }^{1} / C y O$ (BDSC \#24644),

421 LexAop-n-syb::spGFP 1-10, UAS-CD4::spGFP ${ }_{11}$ (BDSC \#64314); UAS-n-syb::spGFP 1-10, LexAop-

422 CD4::spGFP 11 (BDSC \#64315), and the TRiP RNAi strains: JF01945 (BDSC \#25925), JF03208 
424 \#42508) and JF01879 (BDSC \#25858), to knock down Dh31-R, Dh44-R1, Dh44-R2, sNPF-R,

425 Pdfr and FMRFaR, respectively.

\section{PQ and PACAP application and survival score}

427 PQ and PACAP treatments were performed on 9 to 11-day-old adult females by direct

428 application of the drug and neuropeptide onto the exposed VNC of decapitated flies as previously

429 described (39). Briefly, flies were anaesthetized on ice for $10 \mathrm{~min}$ and their heads were cut off

430 with 7-mm blade spring scissors (Fine Science tools). 30 decapitated flies per condition were

431 transferred to a 2-inch Petri dish (10 flies per dish) and allowed to recover for a few minutes until

432 they stood on their legs. A $5 \mu$ droplet of PQ (methyl viologen dichloride hydrate; Sigma-

433 Aldrich 856177) diluted in Drosophila Ringer's solution (in mM: $130 \mathrm{NaCl}, 4.7 \mathrm{KCl}, 1.8 \mathrm{CaCl}_{2}$,

$4340.5 \mathrm{Na}_{2} \mathrm{HPO}_{4}, 0.35 \mathrm{KH}_{2} \mathrm{PO}_{4}, \mathrm{pH} 7.4$ adjusted with $150 \mathrm{Na}_{2} \mathrm{HPO}_{4}$ ) or Ringer's only (R) for

435 controls, was applied for $5 \mathrm{~s}$ with a P10 Pipetman. The same droplet was successively used for 10

436 flies. A concentration of $80 \mathrm{mM}$ PQ was generally used that gave 30-40\% survival of wild type

437 flies after $2 \mathrm{~h}$ (39). Flies were considered as dead when they laid on the side or back and did not

438 react to a slight mechanical stimulus on the legs. Survival rate was monitored every $30 \mathrm{~min}$. Each

439 experiment was repeated independently at least twice. The same procedure was used for DA

440 application to the VNC except that DA concentration in the droplet was $35 \mathrm{mM}$, as previously

441 described (39). For PACAP, PACAP-6-38 and PDF pretreatments, a droplet containing the

442 neuropeptide at $2 \mu \mathrm{M}$ (precisely $2.2 \mu \mathrm{M}$, i.e. 10, 8.9 and $4.3 \mu \mathrm{g} / \mathrm{ml}$, respectively), in Ringer's, or

$44320 \mu \mathrm{M}$ for PDF in some experiments, was applied to the decapitated flies the same way 30 min

444 prior to the exposure to PQ. The 38-amino acid form of PACAP (His-Ser-Asp-Gly-Ile-Phe-Thr-

445 Asp-Ser-Tyr-Ser-Arg-Tyr-Arg-Lys-Gln-Met-Ala-Val-Lys-Lys-Tyr-Leu-Ala-Ala-Val-Leu-Gly- 
446 Lys-Arg-Tyr-Lys-Gln-Arg-Val-Lys-Asn-Lys-NH2) was synthesized by solid-phase methodology

447 as previously described (115). PACAP-6-38 was purchased from Tocris Bioscience, Bristol, UK,

448 and Drosophila PDF (Asn-Ser-Glu-Leu-Ile-Asn-Ser-Leu-Leu-Ser-Leu-Pro-Lys-Asn-Met-Asn-

449 Asp-Ala-NH2) from NovoPro Bioscience Inc., Shanghai, China.

450 Detection of caspase activity

451 We have used the CaspaseTracker biosensor system to detect caspase activity in VNC cells of

452 adult flies exposed to PQ. This system is composed of two genetic components: a biosensor

453 encoding the Gal4 transcription factor sequestered in the cytoplasm by a caspase-cleavable

454 membrane anchor (59), and the G-TRACE fluorescent protein system (116). Following caspase

455 activation, Gal4 is released from its membrane anchor, translocates to the nucleus and drives the

456 expression of both cytosolic red fluorescent protein (RFP), indicating recent or on-going caspase

457 activity, and FLP recombinase, which induces permanent expression of nucleus-targeted GFP.

458 10-11-day-old CaspaseTracker female flies were pretreated with either Ringer's alone (controls)

459 or $10 \mu \mathrm{g} / \mathrm{ml}$ PACAP in the same solution for $30 \mathrm{~min}$ before applying PQ. After 90 min of PQ

460 treatment, 4 or 5 VNCs per condition were dissected in $\mathrm{Ca}^{2+}$-free Ringer's, fixed in $4 \%$ (wt/vol)

461 paraformaldehyde in phosphate-buffered saline (PBS: $130 \mathrm{mM} \mathrm{NaCl}, 7 \mathrm{mM} \mathrm{Na}_{2} \mathrm{HPO}_{4}, 3 \mathrm{mM}$

$462 \mathrm{KH}_{2} \mathrm{PO}_{4}$ ), washed 3 times in PBS and then mounted for confocal microscopy examination.

\section{ROS measurement}

464 ROS detection in the Drosophila CNS was performed using the dihydroethidium (DHE) dye

465 (Life technologies) following a previously described procedure (117, 118). 10-day-old

466 decapitated female flies were exposed to PQ for 90 min with or without (control) 30-min

467 pretreatment with PACAP. Their VNCs were dissected in Schneider's Insect Medium, and

468 incubated in $30 \mu \mathrm{M} \mathrm{DHE}$ in the same medium for $5 \mathrm{~min}$ in the dark. After 5 min of fixation in 
$7 \%$ formaldehyde in PBS (pH 7, at room temperature), the VNCs were immediately imaged on a

470 confocal microscope, as indicated below. Relative ROS levels were measured by quantification

471 of the average intensity of the dye fluorescence using the Fiji software (119).

\section{Immunohistochemistry and split-GFP reconstitution}

473 Whole-mount VNC immunostaining was performed as previously described $(39,120)$. The

474 primary antibodies were mouse monoclonal anti-GFP ( Sigma-Aldrich G6539, 1:200 or 1:250),

475 rabbit polyclonal anti-PDF (kindly provided by Dr. François Rouyer, 1:100) and rabbit

476 polyclonal anti-TH (Novus Biologicals NB300-109, 1:1000). The secondary antibodies were goat

477 anti-mouse and anti-rabbit conjugated to Alexa Fluor 488 or 555 (Invitrogen Molecular Probes,

$4781: 250$ or 1:1000). For the visualization of potential synaptic connectivity by split-GFP

479 reconstitution (GRASP method) $(74,75)$, the Drosophila lines LexAop-n-syb::spGFP ${ }_{1-10}, U A S$ -

$480 \quad C D 4: \because s p G F P_{11}$ and $U A S-n-s y b:: s p G F P_{1-10}$, LexAop-CD4::spGFP 11 were crossed to the

481 recombined driver line Pdf-Gal4; TH-LexA. The VNCs of 7-10-day-old female flies were

482 dissected and processed for TH and GFP co-immunostaining.

\section{Fluorescence confocal microscopy}

$484 \quad$ VNCs were mounted on slides using as antifade reagent, either ProLong Gold Antifade

485 reagent (ThermoFisher Scientific) for CaspaseTracker staining, or Vectashield (Vector

486 Laboratories) for ROS measurements. Images were acquired with a Nikon A1R confocal

487 microscope. A minimum of 4 or 5 VNCs were scored over at least 3 trials. Laser, filter and gain

488 settings remained constant within each experiment, and channels were scanned sequentially.

489 Confocal Z-stacks were analyzed and processed using the Fiji software.

\section{Statistical analysis}


491 Statistical analyses were performed with Prism 6 (GraphPad Software, La Jolla, CA, USA), 492 using either one-way or two-way ANOVA with Tukey's post-hoc multiple comparison test, or 493 Student's $t$ test. Errors bars represent standard errors of the mean (SEM) of 3 or 4 independent 494 determinations. Statistical significance in all figures: ${ }^{*} p<0.05,{ }^{* *} p<0.01,{ }^{* * *} p<0.001$.

496 Acknowledgments

497 We thank Drs Ronald L. Davis, Paul Taghert, François Rouyer and Sean Sweeney for 498 providing Drosophila stocks, Dr. François Rouyer for the gift of anti-PDF antibody and Dr. Paul 499 Taghert for helpful suggestions. This work was supported by a France-Tunisia CMCU-Campus 500 France/PHC Utique 16G0820 /34940PK exchange program to Olfa Masmoudi-Kouki and David 501 Vaudry, and funding from Fondation de France, PSL Research University, ESPCI Paris and 502 Labex MemoLife (ANR-10-LABX-54 MEMO LIFE) to SB. Funders had no role in study design, 503 data collection and analysis, decision to publish, or preparation of the manuscript. Khadija Hajji's $504 \mathrm{PhD}$ was supported by fellowships from the Tunisian Higher Education Ministry, Tunis 505 University LR18ES03, France-Tunisia exchange program PHC-Utique and the French Société de 506 Neuroendocrinologie.

507

508 Conflict of interest statement

509

510 The authors have no conflict of interest to declare.

511

512

513 


\section{References}

1. Kalia, L.V. and Lang, A.E. (2015) Parkinson's disease. Lancet, 386, 896-912.

2. Ritz, B., Rhodes, S.L., Bordelon, Y. and Bronstein, J. (2012) $\alpha$-Synuclein genetic variants predict faster motor symptom progression in idiopathic Parkinson disease. PLoS. ONE, 7, e36199.

3. Fleming, S.M. (2017) Mechanisms of gene-environment interactions in Parkinson's disease. Curr. Environ. Health. Rep, 4, 192-199.

4. Forno, L.S. (1996) Neuropathology of Parkinson's disease. J. Neuropathol. Exp. Neurol., 55, 259-272.

5. Tanner, C.M., Kamel, F., Ross, G.W., Hoppin, J.A., Goldman, S.M., Korell, M., Marras, C., Bhudhikanok, G.S., Kasten, M., Chade, A.R., et al. (2011) Rotenone, paraquat, and Parkinson's disease. Environ. Health. Perspect., 119, 866-872.

6. Pezzoli, G. and Cereda, E. (2013) Exposure to pesticides or solvents and risk of Parkinson disease. Neurology, 80, 2035-2041.

7. Nandipati, S. and Litvan, I. (2016) Environmental exposures and Parkinson's disease. Int. J. Environ. Res. Public. Health, 13.

8. Brooks, A.I., Chadwick, C.A., Gelbard, H.A., Cory-Slechta, D.A. and Federoff, H.J. (1999) Paraquat elicited neurobehavioral syndrome caused by dopaminergic neuron loss. Brain. Res., 823, $1-10$.

9. Ossowska, K., Wardas, J., Smiałowska, M., Kuter, K., Lenda, T., Wierońska, J.M., Zieba, B., Nowak, P., Dabrowska, J., Bortel, A., et al. (2005) A slowly developing dysfunction of dopaminergic nigrostriatal neurons induced by long-term paraquat administration in rats, an animal model of preclinical stages of Parkinson's disease ? Eur. J. Neurosci., 22, 1294-1304.

10. Peng, J., Stevenson, F.F., Doctrow, S.R. and Andersen, J.K. (2005) Superoxide dismutase/catalase mimetics are neuroprotective against selective paraquat-mediated dopaminergic neuron death in the substantial nigra, implications for Parkinson disease. J. Biol. Chem., 280, 29194-29198.

11. Fernagut, P.O., Hutson, C.B., Fleming, S.M., Tetreaut, N.A., Salcedo, J., Masliah, E. and Chesselet, M.F. (2007) Behavioral and histopathological consequences of paraquat intoxication in mice, effects of alpha-synuclein over-expression. Synapse, 61, 991-1001.

12. Franco, R., Li, S., Rodriguez-Rocha, H., Burns, M. and Panayiotidis, M.I. (2010) Molecular mechanisms of pesticide-induced neurotoxicity, relevance to Parkinson's disease. Chem. Biol. Interact., 188, 289-300.

13. Bus, J.S. and Gibson, J.E. (1984) Paraquat, model for oxidant-initiated toxicity. Environ. Health. Perspect., 55, 37-46. 
14. McCormack, A.L., Thiruchelvam, M., Manning-Bog, A.B., Thiffault, C., Langston, J.W., Cory-Slechta, D.A. and Di Monte, D.A. (2002) Environmental risk factors and Parkinson's disease, selective degeneration of nigral dopaminergic neurons caused by the herbicide paraquat. Neurobiol. Dis., 10, 119-127.

15. Ramachandiran, S., Hansen, J.M., Jones, D.P., Richardson, J.R. and Miller, G.W. (2007) Divergent mechanisms of paraquat, MPP+, and rotenone toxicity, oxidation of thioredoxin and caspase-3 activation. Toxicol. Sci., 95, 163-171.

16. Cochemé, H.M. and Murphy, M.P. (2008) Complex I is the major site of mitochondrial superoxide production by paraquat. J. Biol. Chem., 283, 1786-1798.

17. Cristóvão, A.C., Choi, D.-H., Baltazar, G., Beal, M.F. and Kim, Y.-S. (2009) The role of NADPH oxidase 1-derived reactive oxygen species in paraquat-mediated dopaminergic cell death. Antioxid. Redox Signal., 11, 2105-2118.

18. Hosamani, R. and Muralidhara (2013) Acute exposure of Drosophila melanogaster to paraquat causes oxidative stress and mitochondrial dysfunction. Arch. Insect. Biochem. Physiol., 83, 25-40.

19. Reczek, C.R., Birsoy, K., Kong, H., Martínez-Reyes, I., Wang, T., Gao, P., Sabatini, D.M. and Chandel, N.S. (2017) A CRISPR screen identifies a pathway required for paraquat-induced cell death. Nat. Chem. Biol., 13, 1274-1279.

20. Vaudry, D., Falluel-Morel, A., Bourgault, S., Basille, M., Burel, D., Wurtz, O., Fournier, A., Chow, B.K.C., Hashimoto, H., Galas, L., et al. (2009) Pituitary adenylate cyclase-activating polypeptide and its receptors, 20 years after the discovery. Pharmacol. Rev., 61, 283-357.

21. Seaborn, T., Masmoudi-Kouli, O., Fournier, A., Vaudry, H. and Vaudry, D. (2011) Protective effects of pituitary adenylate cyclase-activating polypeptide (PACAP) against apoptosis. Curr. Pharm. Des., 17, 204-214.

22. Reglodi, D., Renaud, J., Tamas, A., Tizabi, Y., Socías, S.B., Del-Bel, E. and Raisman-Vozari, R. (2017) Novel tactics for neuroprotection in Parkinson's disease, role of antibiotics, polyphenols and neuropeptides. Prog. Neurobiol., 155, 120-148.

23. Wang, G., Pan, J., Tan, Y.-Y., Sun, X.-K., Zhang, Y.-F., Zhou, H.-Y., Ren, R.-J., Wang, X.-J. and Chen, S.-D. (2008) Neuroprotective effects of PACAP27 in mice model of Parkinson's disease involved in the modulation of $\mathrm{K}(\mathrm{ATP})$ subunits and $\mathrm{D} 2$ receptors in the striatum. Neuropeptides, 42, 267-276.

24. Dejda, A., Seaborn, T., Bourgault, S., Touzani, O., Fournier, A., Vaudry, H. and Vaudry, D. (2011) PACAP and a novel stable analog protect rat brain from ischemia, insight into the mechanisms of action. Peptides, 32, 1207-1216.

25. Watson, M.B., Nobuta, H., Abad, C., Lee, S.K., Bala, N., Zhu, C., Richter, F., Chesselet, M.-F. and Waschek, J.A. (2013) PACAP deficiency sensitizes nigrostriatal dopaminergic neurons to 
paraquat-induced damage and modulates central and peripheral inflammatory activation in mice. Neuroscience, 240, 277-286.

26. Han, P., Tang, Z., Yin, J., Maalouf, M., Beach, T.G., Reiman, E.M. and Shi, J. (2014) Pituitary adenylate cyclase-activating polypeptide protects against $\beta$-amyloid toxicity. Neurobiol. Aging, 35, 2064-2071.

27. Lamine, A., Létourneau, M., Doan, N.D., Maucotel, J., Couvineau, A., Vaudry, H., Chatenet, D., Vaudry, D. and Fournier, A. (2016) Characterizations of a synthetic pituitary adenylate cyclase-activating polypeptide analog displaying potent neuroprotective activity and reduced in vivo cardiovascular side effects in a Parkinson's disease model. Neuropharmacology, 108, 440450 .

28. Peng, J., Stevenson, F.F., Oo, M.L. and Andersen, J.K. (2009) Iron-enhanced paraquatmediated dopaminergic cell death due to increased oxidative stress as a consequence of microglial activation. Free Radic. Biol. Med., 46, 312-320.

29. Pandey, U.B. and Nichols, C.D. (2011) Human disease models in Drosophila melanogaster and the role of the fly in therapeutic drug discovery. Pharmacol. Rev., 63, 411-436.

30. Jaiswal, M., Sandoval, H., Zhang, K., Bayat, V. and Bellen, H.J. (2012) Probing mechanisms that underlie human neurodegenerative diseases in Drosophila. Annu. Rev. Genet., 46, 371-396.

31. Fernández-Hernández, I., Scheenaard, E., Pollarolo, G. and Gonzalez, C. (2016) The translational relevance of Drosophila in drug discovery. EMBO. Rep., 17, 471-472.

32. Perrimon, N., Bonini, N.M. and Dhillon, P. (2016) Fruit flies on the front line, the translational impact of Drosophila. Dis. Model. Mech, 9, 229-231.

33. Coulom, H. and Birman, S. (2004) Chronic exposure to rotenone models sporadic Parkinson's disease in Drosophila melanogaster. J. Neurosci., 24, 10993-10998.

34. Chaudhuri, A., Bowling, K., Funderburk, C., Lawal, H., Inamdar, A., Wang, Z. and O’Donnell, J.M. (2007) Interaction of genetic and environmental factors in a Drosophila parkinsonism model. J. Neurosci., 27, 2457-2467.

35. Bayersdorfer, F., Voigt, A., Schneuwly, S. and Botella, J.A. (2010) Dopamine-dependent neurodegeneration in Drosophila models of familial and sporadic Parkinson's disease. Neurobiol. Dis., 40, 113-119.

36. Islam, R., Yang, L., Sah, M., Kannan, K., Anamani, D., Vijayan, C., Kwok, J., Cantino, M.E., Beal, M.F. and Fridell, Y.-W.C. (2012) A neuroprotective role of the human uncoupling protein 2 (hUCP2) in a Drosophila Parkinson's disease model. Neurobiol. Dis., 46, 137-146.

37. Martin, C.A., Barajas, A., Lawless, G., Lawal, H.O., Assani, K., Lumintang, Y.P., Nunez, V. and Krantz, D.E. (2014) Synergistic effects on dopamine cell death in a Drosophila model of chronic toxin exposure. Neurotoxicology, 44, 344-351. 
38. Varga, S.J., Qi, C., Podolsky, E. and Lee, D. (2014) A new Drosophila model to study the interaction between genetic and environmental factors in Parkinson's disease. Brain. Res., 1583, $277-286$.

39. Cassar, M., Issa, A.-R., Riemensperger, T., Petitgas, C., Rival, T., Coulom, H., Iché-Torres, M., Han, K.-A. and Birman, S. (2015) A dopamine receptor contributes to paraquat-induced neurotoxicity in Drosophila. Hum. Mol. Genet., 24, 197-212.

40. Shukla, A.K., Pragya, P., Chaouhan, H.S., Tiwari, A.K., Patel, D.K., Abdin, M.Z. and Chowdhuri, D.K. (2014) Heat shock protein-70 (Hsp-70) suppresses paraquat-induced neurodegeneration by inhibiting JNK and caspase-3 activation in Drosophila model of Parkinson's disease. PLoS. ONE, 9, e98886.

41. Nässel, D.R. and Winther, A.M.E. (2010) Drosophila neuropeptides in regulation of physiology and behavior. Prog. Neurobiol., 92, 42-104.

42. Taghert, P.H. and Nitabach, M.N. (2012) Peptide neuromodulation in invertebrate model systems. Neuron, 76, 82-97.

43. Feany, M.B. and Quinn, W.G. (1995) A neuropeptide gene defined by the Drosophila memory mutant amnesiac. Science, 268, 869-873.

44. Hashimoto, H., Shintani, N. and Baba, A. (2002) Higher brain functions of PACAP and a homologous Drosophila memory gene amnesiac, insights from knockouts and mutants. Biochem. Biophys. Res. Commun., 297, 427-431.

45. Pirger, Z., Krajcs, N. and Kiss, T. (2016) Occurrence, distribution, and physiological function of pituitary adenylyl cyclase-activating polypeptide in invertebrate species. In Pituitary Adenylate Cyclase Activating Polypeptide - PACAP, current topics in neurotoxicity. Springer, Cham, pp. 19-31.

46. Kiss, T. and Pirger, Z. (2013) Multifunctional role of PACAP-like peptides in molluscs. Protein. Pept. Lett., 20, 628-635.

47. Zhong, Y. and Peña, L.A. (1995) A novel synaptic transmission mediated by a PACAP-like neuropeptide in Drosophila. Neuron, 14, 527-536.

48. Bhattacharya, A., Lakhman, S.S. and Singh, S. (2004) Modulation of L-type calcium channels in Drosophila via a pituitary adenylyl cyclase-activating polypeptide (PACAP)-mediated pathway. J. Biol. Chem., 279, 37291-37297.

49. Hyun, S., Lee, Y., Hong, S.-T., Bang, S., Paik, D., Kang, J., Shin, J., Lee, J., Jeon, K., Hwang, S., et al. (2005) Drosophila GPCR Han is a receptor for the circadian clock neuropeptide PDF. Neuron, 48, 267-278.

50. Lear, B.C., Merrill, C.E., Lin, J.-M., Schroeder, A., Zhang, L. and Allada, R. (2005) A G 
protein-coupled receptor, groom-of-PDF, is required for PDF neuron action in circadian behavior. Neuron, 48, 221-227.

51. Mertens, I., Vandingenen, A., Johnson, E.C., Shafer, O.T., Li, W., Trigg, J.S., De Loof, A., Schoofs, L. and Taghert, P.H. (2005) PDF receptor signaling in Drosophila contributes to both circadian and geotactic behaviors. Neuron, 48, 213-219.

52. Mertens, I., Husson, S.J., Janssen, T., Lindemans, M. and Schoofs, L. (2007) PACAP and PDF signaling in the regulation of mammalian and insect circadian rhythms. Peptides, 28, 1775-1783.

53. Golombek, D.A. and Rosenstein, R.E. (2010) Physiology of circadian entrainment. Physiol. Rev., 90, 1063-1102.

54. Fifel, K. (2017) Alterations of the circadian system in Parkinson's disease patients. Mov. Disord., 32, 682-692.

55. Hood, S. and Amir, S. (2017) Neurodegeneration and the circadian clock. Front. Aging. Neurosci, 9, 170.

56. Torres, G. and Horowitz, J.M. (1998) Activating properties of cocaine and cocaethylene in a behavioral preparation of Drosophilamelanogaster. Synapse, 29, 148-161.

57. Yellman, C., Tao, H., He, B. and Hirsh, J. (1997) Conserved and sexually dimorphic behavioral responses to biogenic amines in decapitated Drosophila. Proc. Natl. Acad. Sci. U.S.A., 94, 4131-4136.

58. Robberecht, P., Gourlet, P., De Neef, P., Woussen-Colle, M.C., Vandermeers-Piret, M.C., Vandermeers, A. and Christophe, J. (1992) Structural requirements for the occupancy of pituitary adenylate-cyclase-activating-peptide (PACAP) receptors and adenylate cyclase activation in human neuroblastoma NB-OK-1 cell membranes. Discovery of PACAP (6-38) as a potent antagonist. Eur. J. Biochem., 207, 239-246.

59. Tang, H.L., Tang, H.M., Fung, M.C. and Hardwick, J.M. (2015) In vivo CaspaseTracker biosensor system for detecting anastasis and non-apoptotic caspase activity. Sci. Rep, 5, 9015.

60. Im, S.H. and Taghert, P.H. (2010) PDF receptor expression reveals direct interactions between circadian oscillators in Drosophila. J. Comp. Neurol., 518, 1925-1945.

61. Krupp, J.J., Billeter, J.-C., Wong, A., Choi, C., Nitabach, M.N. and Levine, J.D. (2013)

Pigment-dispersing factor modulates pheromone production in clock cells that influence mating in Drosophila.Neuron, 79, 54-68.

62. Emery, P., So, W.V., Kaneko, M., Hall, J.C. and Rosbash, M. (1998) CRY, a Drosophila clock and light-regulated cryptochrome, is a major contributor to circadian rhythm resetting and photosensitivity. Cell, 95, 669-679.

63. Kaneko, M. and Hall, J.C. (2000) Neuroanatomy of cells expressing clock genes in 
Drosophila, transgenic manipulation of the period and timeless genes to mark the perikarya of circadian pacemaker neurons and their projections. J. Comp. Neurol., 422, 66-94.

64. Hamasaka, Y. and Nässel, D.R. (2006) Mapping of serotonin, dopamine, and histamine in relation to different clock neurons in the brain of Drosophila. J. Comp. Neurol., 494, 314-330.

65. Shafer, O.T. and Taghert, P.H. (2009) RNA-interference knockdown of Drosophila pigment dispersing factor in neuronal subsets, the anatomical basis of a neuropeptide's circadian functions. PLoS ONE, 4, e8298.

66. Renn, S.C., Park, J.H., Rosbash, M., Hall, J.C. and Taghert, P.H. (1999) A pdf neuropeptide gene mutation and ablation of PDF neurons each cause severe abnormalities of behavioral circadian rhythms in Drosophila.Cell, 99, 791-802.

67. Huang, W., Lyman, R.F., Lyman, R.A., Carbone, M.A., Harbison, S.T., Magwire, M.M. and Mackay, T.F. (2016) Spontaneous mutations and the origin and maintenance of quantitative genetic variation. Elife, 5.

68. Nitabach, M.N., Sheeba, V., Vera, D.A., Blau, J. and Holmes, T.C. (2005) Membrane electrical excitability is necessary for the free-running larval Drosophila circadian clock. $J$. Neurobiol., 62, 1-13.

69. Choi, C., Cao, G., Tanenhaus, A.K., McCarthy, E.V., Jung, M., Schleyer, W., Shang, Y., Rosbash, M., Yin, J.C.P. and Nitabach, M.N. (2012) Autoreceptor control of peptide/neurotransmitter corelease from PDF neurons determines allocation of circadian activity in Drosophila. Cell. Rep, 2, 332-344.

70. Beckwith, E.J. and Ceriani, M.F. (2015) Communication between circadian clusters, the key to a plastic network. FEBS. Lett., 589, 3336-3342.

71. Shafer, O.T., Kim, D.J., Dunbar-Yaffe, R., Nikolaev, V.O., Lohse, M.J. and Taghert, P.H. (2008) Widespread receptivity to neuropeptide PDF throughout the neuronal circadian clock network of Drosophila revealed by real-time cyclic AMP imaging. Neuron, 58, 223-237.

72. Park, J.H., Helfrich-Förster, C., Lee, G., Liu, L., Rosbash, M. and Hall, J.C. (2000)

Differential regulation of circadian pacemaker output by separate clock genes in Drosophila. Proc. Natl. Acad. Sci. U.S.A., 97, 3608-3613.

73. Friggi-Grelin, F., Coulom, H., Meller, M., Gomez, D., Hirsh, J. and Birman, S. (2003) Targeted gene expression in Drosophila dopaminergic cells using regulatory sequences from tyrosine hydroxylase. J. Neurobiol., 54, 618-627.

74. Feinberg, E.H., Vanhoven, M.K., Bendesky, A., Wang, G., Fetter, R.D., Shen, K. and Bargmann, C.I. (2008) GFP Reconstitution Across Synaptic Partners (GRASP) defines cell contacts and synapses in living nervous systems. Neuron, 57, 353-363.

75. Macpherson, L.J., Zaharieva, E.E., Kearney, P.J., Alpert, M.H., Lin, T.-Y., Turan, Z., Lee, C.- 
H. and Gallio, M. (2015) Dynamic labelling of neural connections in multiple colours by transsynaptic fluorescence complementation. Nat. Commun, 6, 10024.

76. Journot, L., Villalba, M. and Bockaert, J. (1998) PACAP-38 protects cerebellar granule cells from apoptosis. Ann. N. Y. Acad. Sci., 865, 100-110.

77. Masmoudi-Kouki, O., Douiri, S., Hamdi, Y., Kaddour, H., Bahdoudi, S., Vaudry, D., Basille, M., Leprince, J., Fournier, A., Vaudry, H., et al. (2011) Pituitary adenylate cyclase-activating polypeptide protects astroglial cells against oxidative stress-induced apoptosis. J. Neurochem., 117, 403-411.

78. Waschek, J.A. (2013) VIP and PACAP, neuropeptide modulators of CNS inflammation, injury, and repair. Br. J. Pharmacol., 169, 512-523.

79. Lee, E.H. and Seo, S.R. (2014) Neuroprotective roles of pituitary adenylate cyclase-activating polypeptide in neurodegenerative diseases. BMB. Rep, 47, 369-375.

80. Douiri, S., Bahdoudi, S., Hamdi, Y., Cubì, R., Basille, M., Fournier, A., Vaudry, H., Tonon, M.-C., Amri, M., Vaudry, D., et al. (2016) Involvement of endogenous antioxidant systems in the protective activity of pituitary adenylate cyclase-activating polypeptide against hydrogen peroxide-induced oxidative damages in cultured rat astrocytes. J. Neurochem., 137, 913-930.

81. Reglodi, D., Tamas, A., Jungling, A., Vaczy, A., Rivnyak, A., Fulop, B.D., Szabo, E., Lubics, A. and Atlasz, T. (2018) Protective effects of pituitary adenylate cyclase activating polypeptide against neurotoxic agents. Neurotoxicology, 66, 185-194.

82. Maasz, G., Zrinyi, Z., Reglodi, D., Petrovics, D., Rivnyak, A., Kiss, T., Jungling, A., Tamas, A. and Pirger, Z. (2017) Pituitary adenylate cyclase-activating polypeptide (PACAP) has a neuroprotective function in dopamine-based neurodegeneration in rat and snail parkinsonian models. Dis. Model. Mech, 10, 127-139.

83. Heimesaat, M.M., Dunay, I.R., Schulze, S., Fischer, A., Grundmann, U., Alutis, M., Kühl, A.A., Tamas, A., Toth, G., Dunay, M.P., et al. (2014) Pituitary adenylate cyclase-activating polypeptide ameliorates experimental acute ileitis and extra-intestinal sequelae. PLoS ONE, 9 , e108389.

84. Szakaly, P., Kiss, P., Lubics, A., Magyarlaki, T., Tamas, A., Racz, B., Lengvari, I., Toth, G. and Reglodi, D. (2008) Effects of PACAP on survival and renal morphology in rats subjected to renal ischemia/reperfusion. $J$. Mol. Neurosci., 36, 89-96.

85. Gray, S.L., Yamaguchi, N., Vencová, P. and Sherwood, N.M. (2002) Temperature-sensitive phenotype in mice lacking pituitary adenylate cyclase-activating polypeptide. Endocrinology, 143, 3946-3954.

86. Mori, H., Nakamachi, T., Ohtaki, H., Yofu, S., Sato, A., Endo, K., Iso, Y., Suzuki, H., Takeyama, Y., Shintani, N., et al. (2010) Cardioprotective effect of endogenous pituitary adenylate cyclase-activating polypeptide on doxorubicin-induced cardiomyopathy in mice. Circ. 
$J .$, 74, 1183-1190.

87. Shukla, A.K., Ratnasekhar, C., Pragya, P., Chaouhan, H.S., Patel, D.K., Chowdhuri, D.K. and Mudiam, M.K.R. (2016) Metabolomic analysis provides insights on paraquat-induced Parkinsonlike symptoms in Drosophila melanogaster. Mol. Neurobiol., 53, 254-269.

88. Miyamoto, K., Tsumuraya, T., Ohtaki, H., Dohi, K., Satoh, K., Xu, Z., Tanaka, S., Murai, N., Watanabe, J., Sugiyama, K., et al. (2014) PACAP38 suppresses cortical damage in mice with traumatic brain injury by enhancing antioxidant activity. J. Mol. Neurosci., 54, 370-379.

89. Mehdi, S.H. and Qamar, A. (2013) Paraquat-induced ultrastructural changes and DNA damage in the nervous system is mediated via oxidative-stress-induced cytotoxicity in Drosophila melanogaster. Toxicol. Sci., 134, 355-365.

90. Vaudry, D., Gonzalez, B.J., Basille, M., Pamantung, T.F., Fontaine, M., Fournier, A. and Vaudry, H. (2000) The neuroprotective effect of pituitary adenylate cyclase-activating polypeptide on cerebellar granule cells is mediated through inhibition of the CED3-related cysteine protease caspase-3/CPP32. Proc. Natl. Acad. Sci. U.S.A., 97, 13390-13395.

91. Pirger, Z., Nemeth, J., Hiripi, L., Toth, G., Kiss, P., Lubics, A., Tamas, A., Hernadi, L., Kiss, T. and Reglodi, D. (2008) PACAP has anti-apoptotic effect in the salivary gland of an invertebrate species, Helix pomatia. J. Mol. Neurosci., 36, 105-114.

92. Peng, J., Mao, X.O., Stevenson, F.F., Hsu,M. and Andersen, J.K. (2004) The herbicide paraquat induces dopaminergic nigral apoptosis through sustained activation of the JNK pathway. J. Biol. Chem., 279, 32626-32632.

93. Fei, Q., McCormack, A.L., Di Monte, D.A. and Ethell, D.W. (2008) Paraquat neurotoxicity is mediated by a Bak-dependent mechanism. J. Biol. Chem., 283, 3357-3364.

94. Dejda, A., Jolivel, V., Bourgault, S., Seaborn, T., Fournier, A., Vaudry, H. and Vaudry, D. (2008) Inhibitory effect of PACAP on caspase activity in neuronal apoptosis, a better understanding towards therapeutic applications in neurodegenerative diseases. J. Mol. Neurosci., 36, 26-37.

95. Li, Y., Guo, F., Shen, J. and Rosbash, M. (2014) PDF and cAMP enhance PER stability in Drosophila clock neurons. Proc. Natl. Acad. Sci. U.S.A., 111, E1284-1290.

96. Seluzicki, A., Flourakis, M., Kula-Eversole, E., Zhang, L., Kilman, V. and Allada, R. (2014) Dual PDF signaling pathways reset clocks via TIMELESS and acutely excite target neurons to control circadian behavior. PLoS. Biol., 12, e1001810.

97. Vecsey, C.G., Pírez, N. and Griffith, L.C. (2014) The Drosophila neuropeptides PDF and sNPF have opposing electrophysiological and molecular effects on central neurons. $J$.

Neurophysiol., 111, 1033-1045.

98. Yao, Z. and Shafer, O.T. (2014) The Drosophila circadian clock is a variably coupled network 
of multiple peptidergic units. Science, 343, 1516-1520.

99. Choi, C., Fortin, J.-P., McCarthy, E. v, Oksman, L., Kopin, A.S. and Nitabach, M.N. (2009) Cellular dissection of circadian peptide signals with genetically encoded membrane-tethered ligands. Curr. Biol., 19, 1167-1175.

100. Agrawal, T., Sadaf, S. and Hasan, G. (2013) A genetic RNAi screen for $\mathrm{IP}_{3} / \mathrm{Ca}^{2+}$ coupled GPCRs in Drosophila identifies the PdfR as a regulator of insect flight. PLoS. Genet., 9, e1003849.

101. Liang, X., Holy, T.E. and Taghert, P.H. (2017) A series of suppressive signals within the Drosophila circadian neural circuit generates sequential daily outputs. Neuron, 94, 1173-1189

102. Means, J.C., Venkatesan, A., Gerdes, B., Fan, J.-Y., Bjes, E.S. and Price, J.L. (2015) Drosophila spaghetti and doubletime link the circadian clock and light to caspases, apoptosis and tauopathy. PLoS. Genet, 11.

103. Vaccaro, A., Issa, A.-R., Seugnet, L., Birman, S. and Klarsfeld, A. (2017) Drosophila clock is required in brain pacemaker neurons to prevent premature locomotor aging independently of its circadian function. PLoS. Genet., 13, e1006507.

104. Emery, P. (2015) Connecting circadian genes to neurodegenerative pathways in fruit flies. PLoS. Genet., 11, e1005266.

105. He, Q., Wu, B., Price, J.L. and Zhao, Z. (2017) Circadian rhythm neuropeptides in Drosophila, signals for normal circadian function and circadian neurodegenerative disease. Int. J. Mol. Sci, 18.

106. Giebultowicz, J.M. (2018) Circadian regulation of metabolism and healthspan in Drosophila.Free Radic. Biol. Med., 119, 62-68.

107. Helfrich-Förster, C. (1997) Development of pigment-dispersing hormone-immunoreactive neurons in the nervous system of Drosophila melanogaster. J. Comp. Neurol., 380, 335-354.

108. Santos, J.G., Vömel, M., Struck, R., Homberg, U., Nässel, D.R. and Wegener, C. (2007) Neuroarchitecture of peptidergic systems in the larval ventral ganglion of Drosophila melanogaster.PLoS. ONE, 2, e695.

109. Potdar, S. and Sheeba, V. (2018) Wakefulness is promoted during day time by PDFR signalling to dopaminergic neurons in Drosophila melanogaster. eNeuro,5, 1-17.

110. Lin, D.M. and Goodman, C.S. (1994) Ectopic and increased expression of Fasciclin II alters motoneuron growth cone guidance. Neuron, 13, 507-523.

111. Sepp, K.J., Schulte, J. and Auld, V.J. (2001) Peripheral glia direct axon guidance across the CNS/PNS transition zone. Dev. Biol., 238, 47-63. 
112. Berry, J.A., Cervantes-Sandoval, I., Chakraborty, M. and Davis, R.L. (2015) Sleep facilitates memory by blocking dopamine neuron-mediated forgetting. Cell, 161, 1656-1667.

113. Paradis, S., Sweeney, S.T. and Davis, G.W. (2001) Homeostatic control of presynaptic release is triggered by postsynaptic membrane depolarization. Neuron, 30, 737-749.

114. Riemensperger, T., Issa, A.-R., Pech, U., Coulom, H., Nguyễn, M.-V., Cassar, M., Jacquet, M., Fiala, A. and Birman, S. (2013) A single dopamine pathway underlies progressive locomotor deficits in a Drosophila model of Parkinson disease. Cell. Rep, 5, 952-960.

115. Jolivel, V., Basille, M., Aubert, N., de Jouffrey, S., Ancian, P., Le Bigot, J.-F., Noack, P., Massonneau, M., Fournier, A., Vaudry, H., et al. (2009) Distribution and functional characterization of pituitary adenylate cyclase-activating polypeptide receptors in the brain of nonhuman primates. Neuroscience, 160, 434-451.

116. Evans, C.J., Olson, J.M., Ngo, K.T., Kim, E., Lee, N.E., Kuoy, E., Patananan, A.N., Sitz, D., Tran, P., Do, M.-T., et al. (2009) G-TRACE, rapid Gal4-based cell lineage analysis in Drosophila. Nat. Methods, 6, 603-605.

117. Owusu-Ansah, E., Yavari, A. and Banerjee, U. (2008) A protocol for in vivo detection of reactive oxygen species. Protocol. Exchange, 10.1038/nprot.2008.23.

118. Issa, A.-R., Sun, J., Petitgas, C., Mesquita, A., Dulac, A., Robin, M., Mollereau, B., Jenny, A., Chérif-Zahar, B. and Birman, S. (2018) The lysosomal membrane protein LAMP2A promotes autophagic flux and prevents SNCA-induced Parkinson disease-like symptoms in the Drosophila brain. Autophagy, 14, 1898-1910.

119. Schindelin, J., Arganda-Carreras, I., Frise, E., Kaynig, V., Longair, M., Pietzsch, T., Preibisch, S., Rueden, C., Saalfeld, S., Schmid, B., et al. (2012) Fiji, an open-source platform for biological-image analysis. Nature. Methods, 9, 676-682.

120. Riemensperger, T., Isabel, G., Coulom, H., Neuser, K., Seugnet, L., Kume, K., Iché-Torres, M., Cassar, M., Strauss, R., Preat, T., et al. (2011) Behavioral consequences of dopamine deficiency in the Drosophila central nervous system. Proc. Natl. Acad. Sci. U.S.A., 108, 834-839. 


\section{Figures Legend}

Figure 1. PACAP decreases PQ-induced oxidative stress and caspase activation in the Drosophila CNS. (A) Application of $80 \mathrm{mM}$ PQ to the VNC decreased fly survival compared to controls treated with Ringer's $(\mathrm{R})$ solution only $\left(\mathrm{R}+\mathrm{PQ} v s \mathrm{R}+\mathrm{R},{ }^{\# \#} p<0.001\right)$. Pretreatment with $2 \mu \mathrm{M}$ PACAP (P) during 30 min had no effect by itself $(\mathrm{P}+\mathrm{R} v s \mathrm{R}+\mathrm{R})$ but markedly delayed PQinduced lethality. The protective action of PACAP was significant until 3 hours after PQ application (P+PQ vs $\left.\mathrm{R}+\mathrm{PQ},{ }^{* *} p<0.01,{ }^{* * *} p<0.001\right)$. Two-way ANOVA with Tukey's posthoc test. (B, C) Evaluation of ROS levels in the VNC after 90 min of PQ exposure. (B) Flies pretreated with $2 \mu \mathrm{M}$ PACAP (right panel) showed reduced DHE fluorescent staining indicating lower ROS in the VNC than flies exposed to PQ only (left panel). Scale bar: $50 \mu \mathrm{m}$. (C) Quantification of the effect of PACAP on PQ-induced ROS accumulation in the VNC. Student's t test, ${ }^{* *} p<0.01$. (D, E) Effect of PACAP on caspase activation induced by PQ exposure after 90 min, as detected by the CaspaseTracker biosensor system. (D) Pre-application of $2 \mu \mathrm{M}$ PACAP significantly decreased the number of activated caspase-positive VNC cells in decapitated flies treated with PQ (right panel) compared to flies exposed to PQ only (left panel). Scale bar: $50 \mu \mathrm{m}$. (E) Quantification of the effect of PACAP on PQ-induced caspase activation in VNC cells. Student's t test, $* * p<0.01$.

Figure 2. $P d f r$ knock down in all neurons or all clock cells increases PQ resistance. (A) Survival kinetics of PQ-intoxicated elav $>P d f r^{\mathrm{RNAi}}, D c r-2$ flies compared to $e l a v>D c r-2$ controls, pre-treated $(\mathrm{P}+\mathrm{PQ})$ or not (R + PQ) with PACAP. Pdfr downregulation in all neurons strongly mitigated PQinduced lethality (as also shown in Table 1) and the survival of these flies was not further increased by PACAP, in contrast to the elav>Dcr-2 control flies. (B) Overexpression of Pdfr in all 
neurons (elav $>$ Pdfr) did not alter Drosophila PQ susceptibility, as compared to the heterozygous driver $(e l a v /+)$ and effector $(P d f r /+)$ controls. (C) $P d f r$ downregulation in all clock cells with the tim-Gal4 driver (tim $\left.>P d f r^{\mathrm{RNAi}}, D c r-2\right)$ significantly protected flies against PQ compared to the survival rate of $P d f r^{\mathrm{RNAi} /}+;$; $D c r-2 /+$ controls $(\mathrm{R}+\mathrm{PQ})$. No further protection could be observed by PACAP pre-application on the $t i m>P d f r^{\mathrm{RNAi}}, D c r-2$ flies, whereas the $P d f r^{\mathrm{RNAi}} /+; D c r-2 /+$ controls were strongly protected by PACAP (P + PQ). (D) Monitoring the survival of PQ-treated elav $>P d f r^{\mathrm{RNAi}}, D c r-2$ and $t i m>P d f r^{\mathrm{RNAi}}, D c r-2$ flies in a parallel experiment showed similar levels of protection compared to $P d f r^{\mathrm{RNAi}}++; D c r-2 /+$ controls when $P d f r$ was down-regulated in neurons $\left.{ }^{*}\right)$ or in clock cells $\left(^{\#}\right)$. Two-way ANOVA with Tukey's post-hoc test: ${ }^{*} p<0.05,{ }^{*} p<0.01$, ${ }^{* * *} p<0.001,{ }^{\# \#} p<0.001,{ }^{\# \#} p<0.001$, ns: not significant.

Figure 3. Lack of PACAP-induced neuroprotection in $P d f r$ and $P d f$ null mutants. (A) In the absence of PDF receptor (mutant $P d f r^{5304}$ ), PQ resistance was not significantly different(ns) from that of wild-type CS flies ( $\left.P d f r^{5304}, \mathrm{R}+\mathrm{PQ} v s \mathrm{CS}, \mathrm{R}+\mathrm{PQ}\right)$, and no protective effect of PACAP against PQ could be observed in these mutant flies ( $\left.P d f r^{5304}, \mathrm{P}+\mathrm{PQ} v s \mathrm{R}+\mathrm{PQ}\right)$. (B) Without expressed PDF neuropeptide (mutant $P d f^{\oplus 1}$ ), flies were also not more resistant to PQ than wild type, and again PACAP did not protect against PQ (ns). (C) The hyperpolarization of PDFreleasing neurons ( $P d f>$ Kir2.1 flies) had no effect in normal conditions (Ringer, R) but increased PQ resistance at 60 and 90 min of exposure compared to driver (PDF/+) and effector (Kir2.1/+) controls. (D) Direct pre-application of $2 \mu \mathrm{M}$ PDF neuropeptide on the VNC of decapitated flies did not affect their survival rate under control (PDF $+\mathrm{R}$ versus $\mathrm{R}+\mathrm{R}$ ) or PQ-intoxicated (PDF + PQ versus R + PQ) conditions (ns). Two-way ANOVA with Tukey's post-hoc test: ${ }^{*} p<0.05$, $* * * p<0.001$, ns: not significant. 
Figure 4. Selective Pdfr knock down in PDF or DA neurons prevents PACAP-mediated neuroprotection. (A) Pdfr downregulation targeted to PDF neurons with the Pdf-Gal4 driver did not protect against PQ but efficiently suppressed PACAP-induced increase in PQ resistance, i.e. the survival of $P d f>P d f r^{\mathrm{RNAi}}, D c r-2$ flies was not different in P + PQ and R + PQ conditions. The control flies $(P d f-G a l 4 /+)$ were in contrast normally protected by a PACAP pre-treatment $(\mathrm{P}+\mathrm{PQ}$ vs R + PQ: $\left.{ }^{* *} p<0.01,{ }^{* * *} p<0.001\right)$. (B) Pdfr knock down selectively in the Abd PDF neurons using Dot-Gal4 also suppressed the protective effect of PACAP and did not increase PQ resistance. The survival of $D o t>P d f r^{\mathrm{RNAi}}, D c r-2$ flies was similar in P+PQ and R+PQ conditions, whereas PACAP pre-treatment fully protected the control flies (Dot-Gal4/+) from PQ toxicity $\left(\mathrm{P}+\mathrm{PQ}\right.$ vs $\left.\mathrm{R}+\mathrm{PQ}:{ }^{*} \mathrm{p}<0.01, * * * \mathrm{p}<0.001\right)$. (C) PDFR downregulation in DA neurons with $T H-$ Gal4 (TH>Pdfr $\left.r^{\mathrm{RNAi}}, \mathrm{Dcr}-2\right)$ similarly prevented PACAP-induced neuroprotection compared to controls (i.e. survival of $T H>P d f r^{\mathrm{RNAi}}, D c r-2$ flies was not significantly different in $\mathrm{P}+\mathrm{PQ}$ and R + PQ conditions at all times) and also did not change PQ susceptibility, whereas the effector control flies $\left(P d f r^{\mathrm{RNAi} /+}\right.$; $\left.D c r-2 /+\right)$ were well protected by PACAP pre-treatment $(\mathrm{P}+\mathrm{PQ} v s \mathrm{R}+$ PQ: $\left.{ }^{*} p<0.05,{ }^{*} p<0.01\right)$. Two-way ANOVA with Tukey’s post-hoc test.

Figure 5. Identification of potential synaptic connections between PDF and DA neurons in the Drosophila VNC by split-GFP reconstitution. The fusion protein n-Syb::spGFP ${ }_{1-10}$, which is targeted to synaptic terminals, was expressed in PDF neurons with $P d f-G a l 4\left(P D F>n S y b:: G F P_{1-}\right.$ 10) and the fusion membrane protein CD4::spGFP ${ }_{11}$ was expressed in DA neurons with $T H-L e x A$ (TH>CD4::spGFP 11 ). TH (magenta) and GFP (green) co-immunostaining. (A) TH immunostaining. (B) GFP immunostaining. (C) Merge. Reconstituted split GFP (rsGFP) fluorescence specifically labelled bilateral axon bundles of DA neurons localized in the abdominal ganglia of the VNC (arrowheads in $\mathbf{B}$ and $\mathbf{C}$ ). Scale bar: $100 \mu \mathrm{m}$. 
Table 1. Effect of neuropeptide receptor downregulation on Drosophila PQ susceptibility

\begin{tabular}{|lcccc|}
\hline Fly genotype & \multicolumn{2}{c}{ elav>Dcr-2 } & \multicolumn{2}{c|}{ elav> $\mathbf{R}^{\mathrm{RNAi}}$, Dcr-2 } \\
\hline Treatment & $\mathbf{P Q}$ & PACAP + PQ & PQ & PACAP + PQ \\
\hline DH31-R & 45 & $85^{* * *}$ & 49 & $84^{* * *}$ \\
\hline DH44-R1 & 54 & $76^{* *}$ & 46 & $62,5^{*}$ \\
\hline DH44-R2 & 34 & $71^{* * *}$ & 45 & $72^{* *}$ \\
\hline SNPF-R & 46 & $75^{* * *}$ & 38 & $71^{* * *}$ \\
\hline FMRFaR & 41 & $65^{* *}$ & 39 & $59^{*}$ \\
\hline Pdfr & 45 & $90^{* * *}$ & $77^{\# \# \#}$ & 82 \\
\hline
\end{tabular}

Genes encoding various neuropeptide GPCRs were knocked down by co-expressing specific interfering double-stranded RNAs $\left(R^{\mathrm{RNAi}}\right)$ and the RNAi booster $D c r-2$ in all neurons with the driver elav-Gal4(elav $>R^{\mathrm{RNAi}}$, Dcr-2flies) or Dcr-2 only for controls (elav $>$ Dcr-2flies). The numbers indicate the percent of surviving flies 90 min after application of PQ without (PQ) or with $(\mathrm{PACAP}+\mathrm{PQ})$ pre-treatment with $2 \mu \mathrm{M}$ PACAP. Statistical significance: PACAP + PQ vs PQ, ${ }^{*} p<0.05,{ }^{* *} p<0.01,{ }^{* * *} p<0.001$; PQ $\left(e l a v>R^{\mathrm{RNAi}}\right.$, Dcr-2)vsPQ $(e l a v>D c r-2),{ }^{\# \#} p<0.001$. One-way ANOVA with Tukey's post-hoc multiple comparison test. 


\section{Abbreviations used}

Clk, Clock; CNS, central nervous system; CS, Canton-S; DA, dopamine; DA neurons, dopaminergic neurons; DHE, dihydroethidium; GFP: green fluorescent protein; GPCR, G proteincoupled receptor; GPX1, glutathione peroxidase 1; GRASP, GFP reconstitution across synaptic partners; GSH, glutathione; MPTP, 1-methyl-4-phenyl-1,2,3,6-tetrahydropyridine; ns: not significant; PACAP, pituitary adenylate cyclase-activating polypeptide; PBS, phosphate-buffered saline; PD, Parkinson's disease; PDF, Pigment dispersing factor; PDFR, PDF receptor; PKA, protein kinase A; PQ, paraquat/1,1'-dimethyl-4-4'-bipyridinium; RFP, red fluorescent protein; RNAi, RNA interference; ROS, reactive oxygen species; rsGFP: reconstituted split GFP; SNpc, substantia nigra pars compacta; SOD, superoxide dismutase; TH, tyrosine hydroxylase; VIP, vasoactive intestinal peptide; VNC, ventral nerve cord; vs: versus. 


\section{Supplementary figure legends}

Figure S1. PACAP protects Drosophila against DA neurotoxicity. Application of $35 \mathrm{mM}$ DA to the exposed VNC of decapitated flies significantly decreased fly survival compared to controls ( $\mathrm{R}$ + DA vs $\mathrm{R}+\mathrm{R}, \# p<0.05, \# \# p<0.01$ ). A 30-min pretreatment with $2 \mu \mathrm{M}$ PACAP prevented DAinduced fly death (P $\left.+\mathrm{DA} v s \mathrm{R}+\mathrm{DA},{ }^{*} p<0.05,{ }^{* * *} p<0.001\right)$. Two-way ANOVA with Tukey's post-hoc test. (Related to Figure 1).

Figure S2. The PACAP receptor antagonist PACAP-6-38 increases Drosophila susceptibility to PQ. Pre-treatment with $2 \mu \mathrm{M}$ PACAP-6-38 (P-6-38) for 30 min significantly accelerated the death of decapitated flies intoxicated by PQ (P-6-38 + PQ vs R + PQ, $* * * p<0.001)$, compared to control flies pre-treated with Ringer's only $(\mathrm{R}+\mathrm{PQ}$ vs $\mathrm{R}+\mathrm{R}$, \#\# $<0.001)$, indicating that the antagonist makes Drosophila more sensitive to oxidative stress. PACAP-6-38 had no effect by itself on fly survival in the absence of PQ (P-6-38+R vs R+R). Two-way ANOVA with Tukey's post-hoc test. (Related to Figure 1).

Figure S3. $P d f r$ downregulation in glial cells does not increase fly PQ resistance. The survival rate of PQ-treated repo $>P d f r^{\mathrm{RNAi}}$; Dcr-2 decapitated flies was not improved compared to $P d f r^{\mathrm{RNAi}} /+$; Dcr-2/+ controls (ns: not significant). (Related to Figure 2).

Figure S4. Expression pattern of the tim-Gal4 driver in the abdominal ganglia. (A, B) Wholemount GFP and PDF co-immunostaining in the VNC of young adult Drosophila expressing membrane-associated GFP under tim-Gal4 control (tim>mCD8::GFP, nSyb::GFP flies). (A) tim- 
Gal4 expresses in neuronal subsets in the abdominal ganglia and in characteristic bilateral axonal bundles (arrows) that may project from dopaminergic cell bodies (see Fig. 5). (B) PDF coimmunostaining shows that tim-Gal4 does not express in the Abd PDF neurons. Scale bar: $50 \mu \mathrm{m}$. (Related to Figure 2 and 5).

Figure S5. No effect of PDF on PQ susceptibility of wild-type flies. Direct application of $20 \mu \mathrm{M}$ PDF to the VNC of decapitated flies did not alter their survival rate under normal (PDF $+\mathrm{R}$ versus $\mathrm{R}+\mathrm{R}$ ) or PQ-intoxicated (PDF + PQ versus $\mathrm{R}+\mathrm{PQ}$ ) conditions (ns: not significant).(Related to Figure 3). 
A

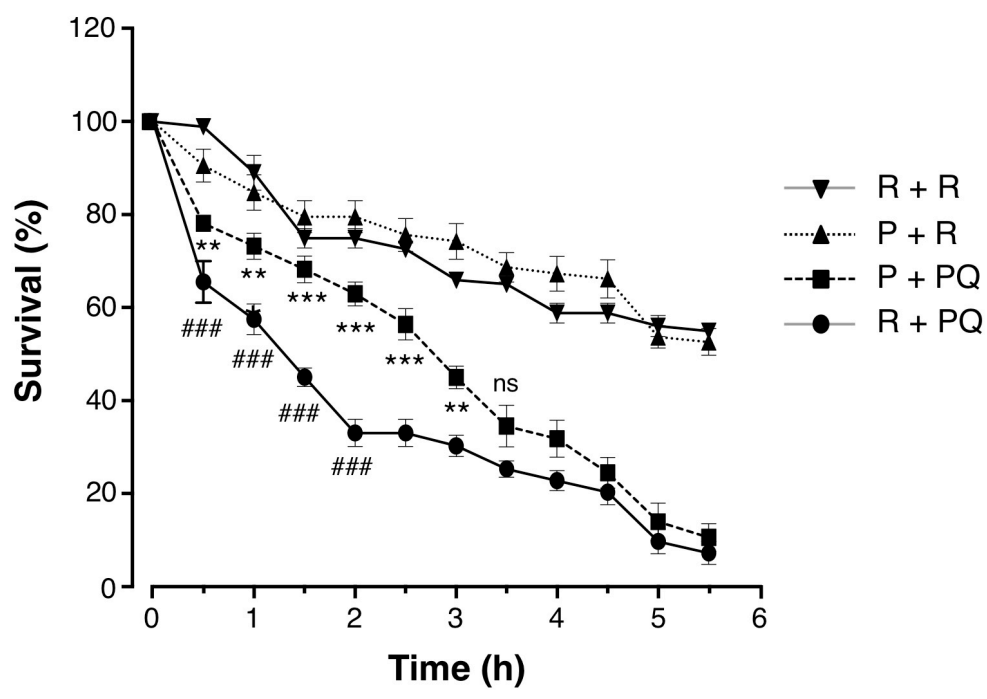

B
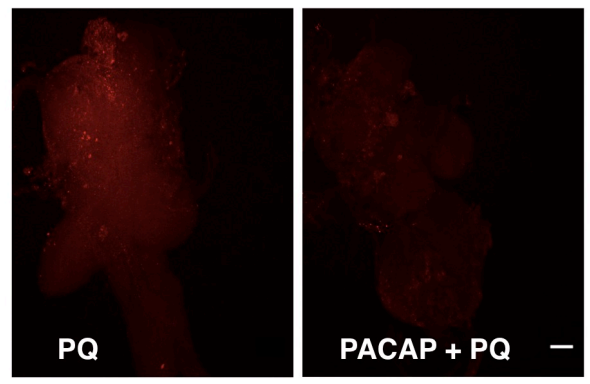

C

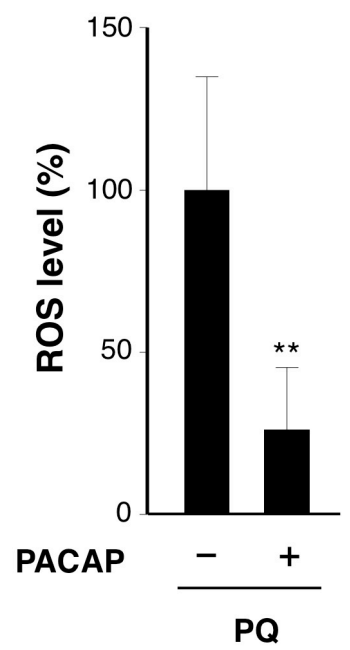

D

E

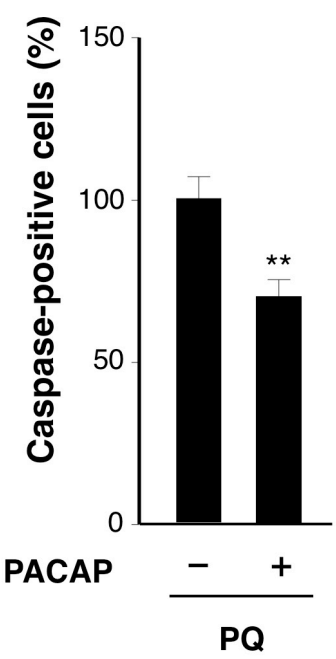

Hajji et al. Figure 1 

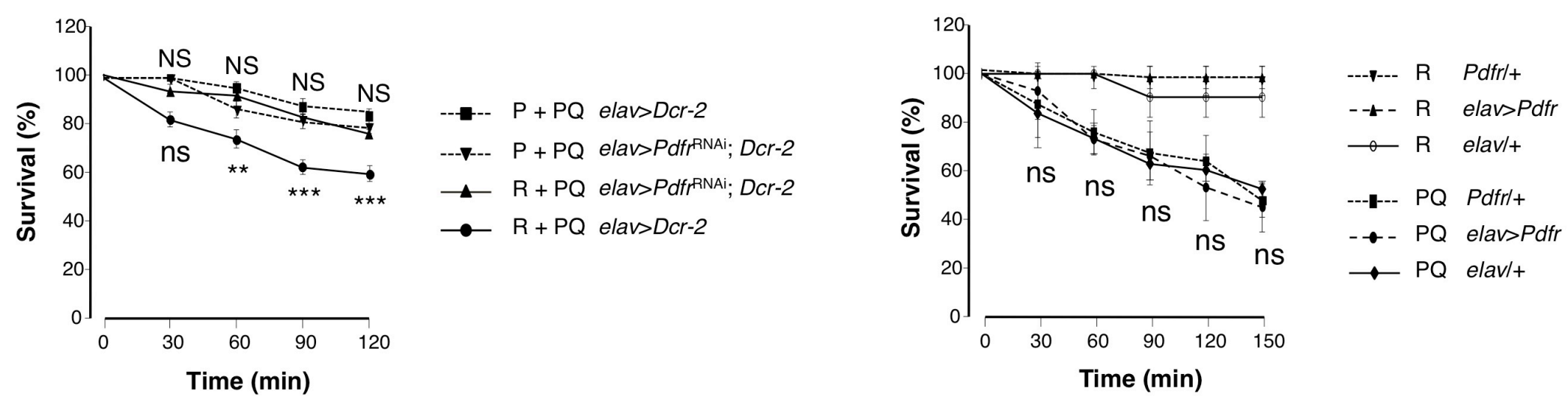

C

D
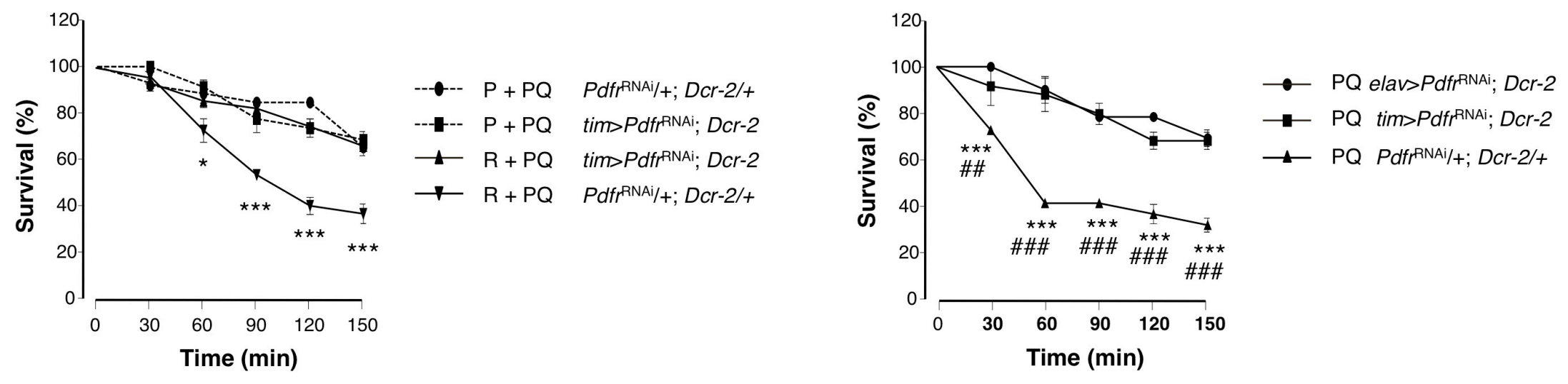

Hajji et al. Figure 2 
A

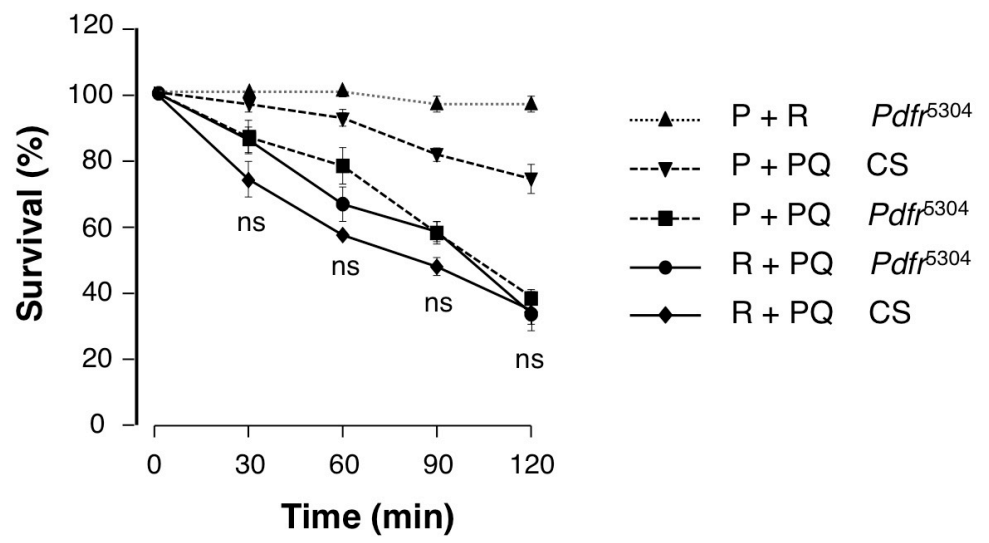

C

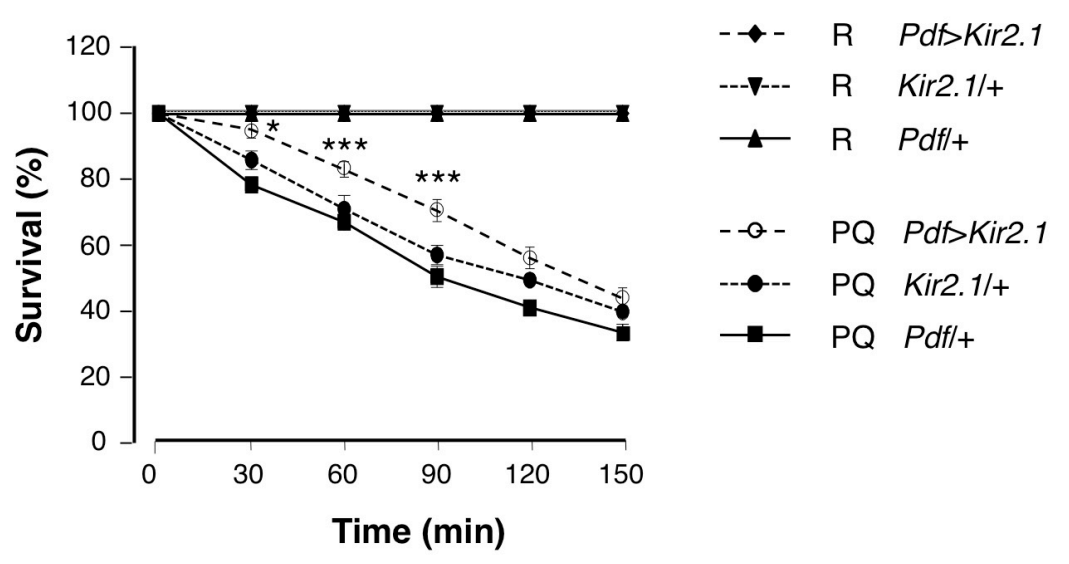

B

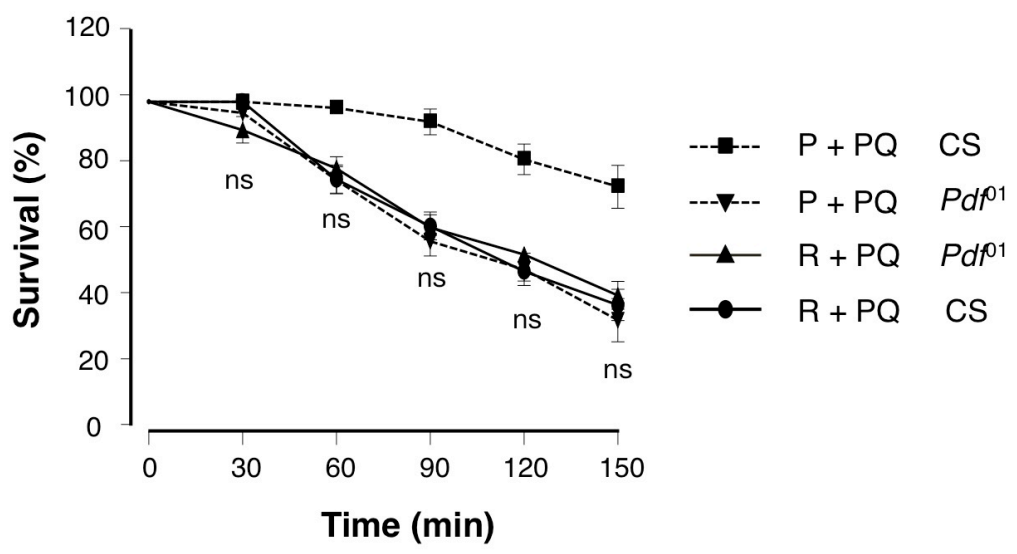

D

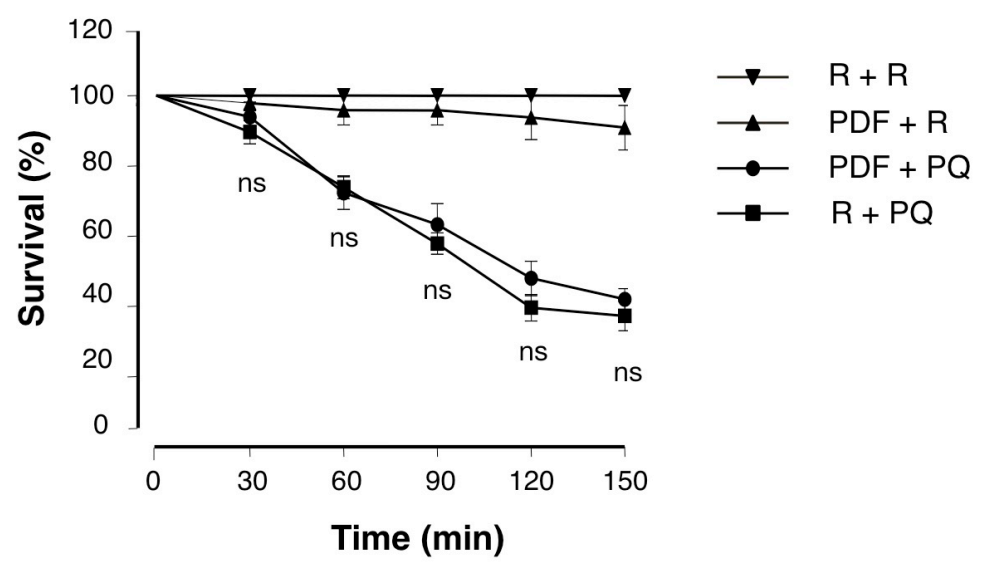

Hajji et al. Figure 3 
A

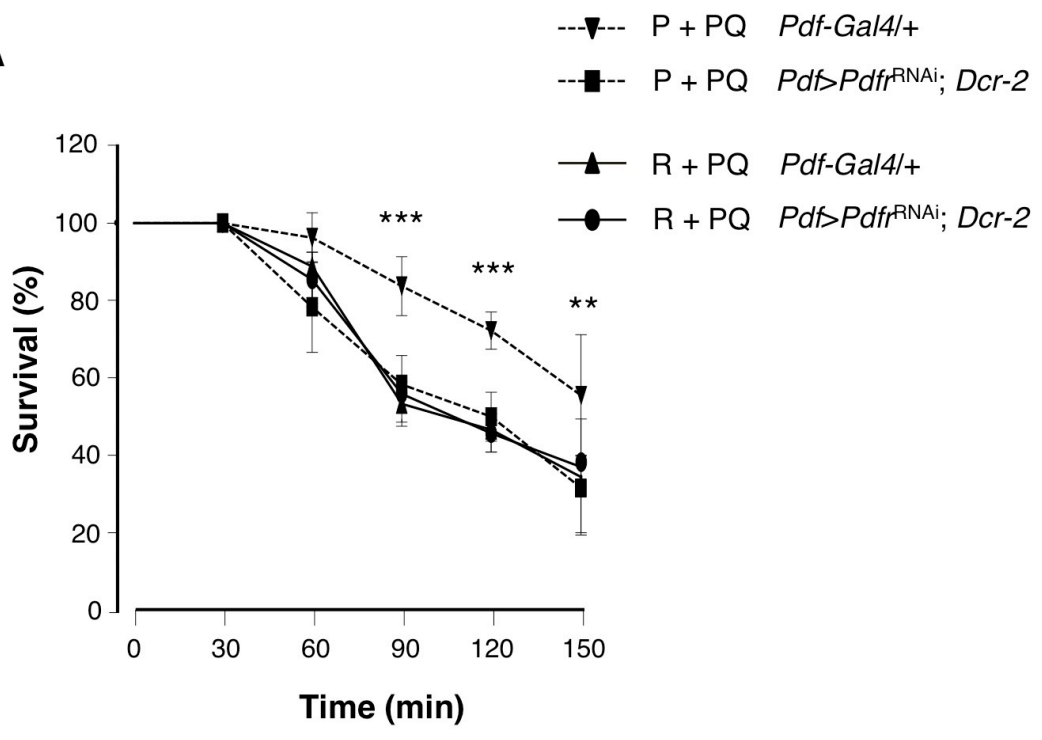

B

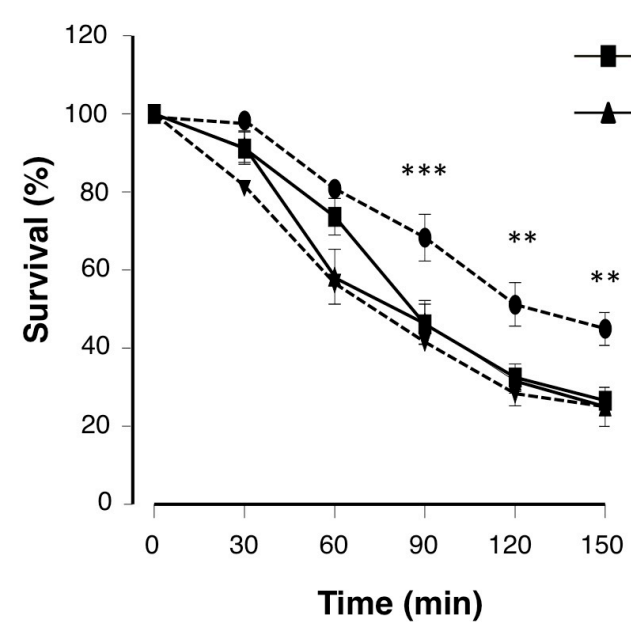

C

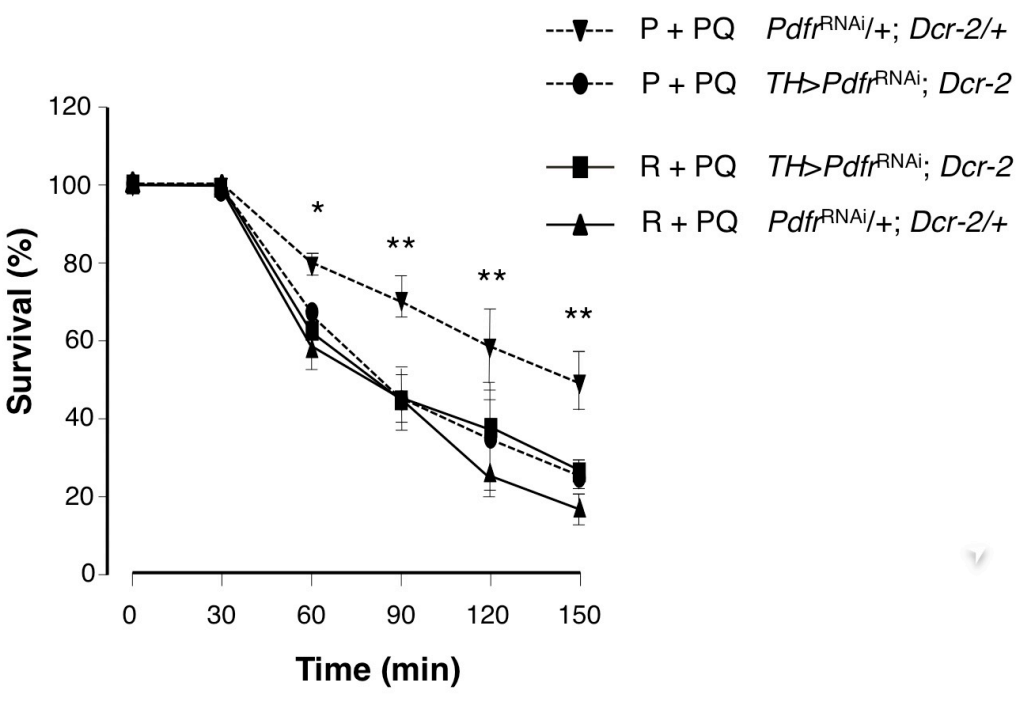

Hajji et al. Figure 4 


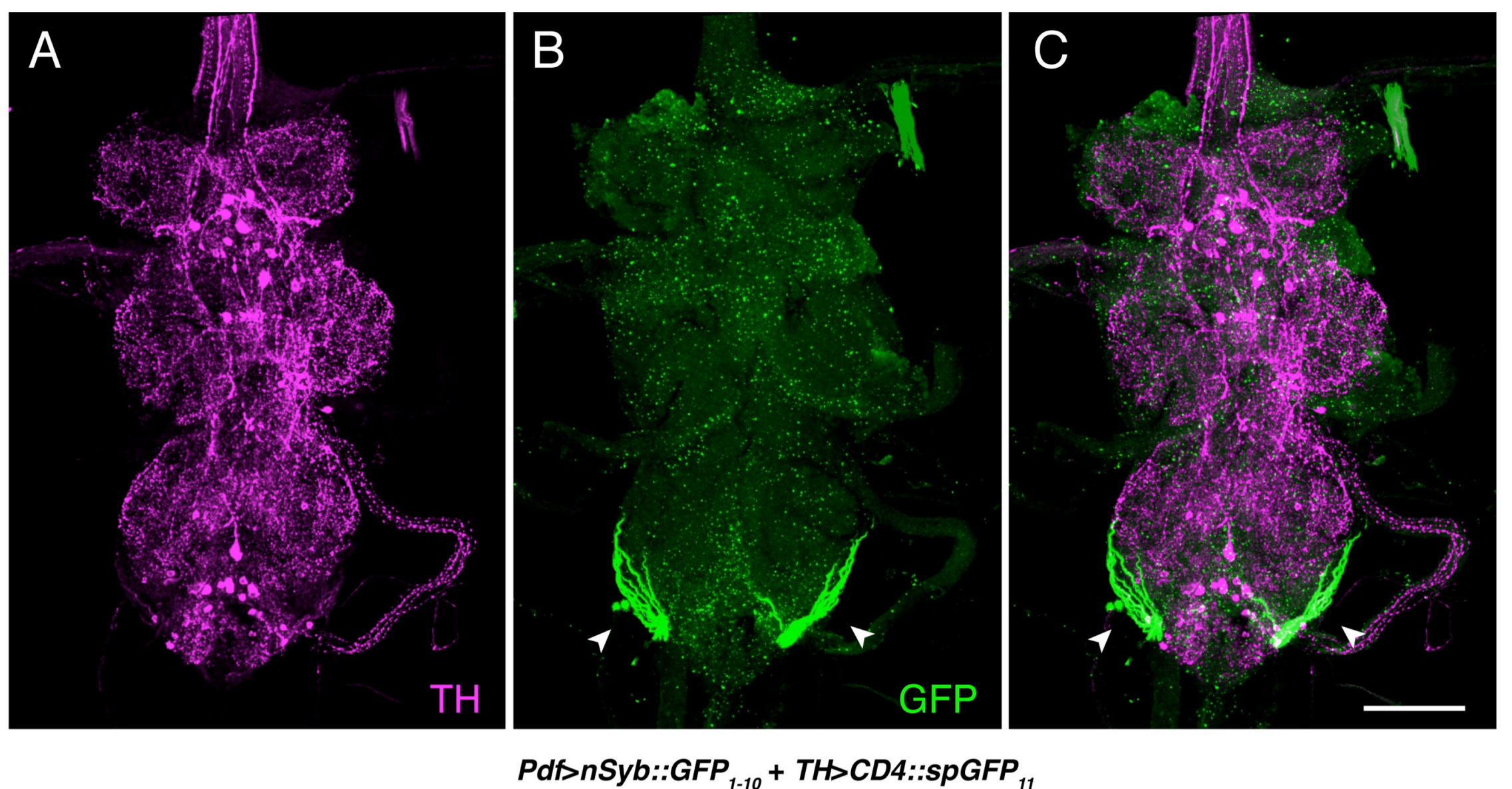




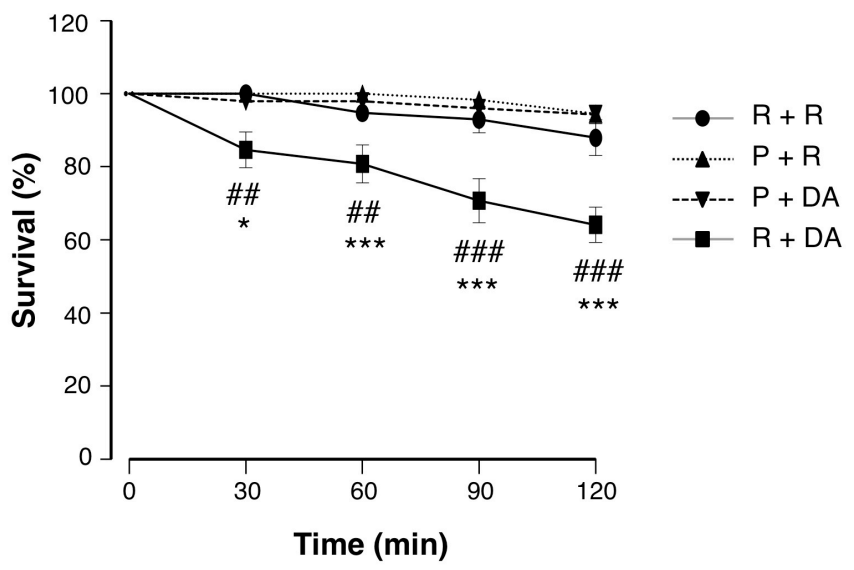

Hajji et al. Figure S1 


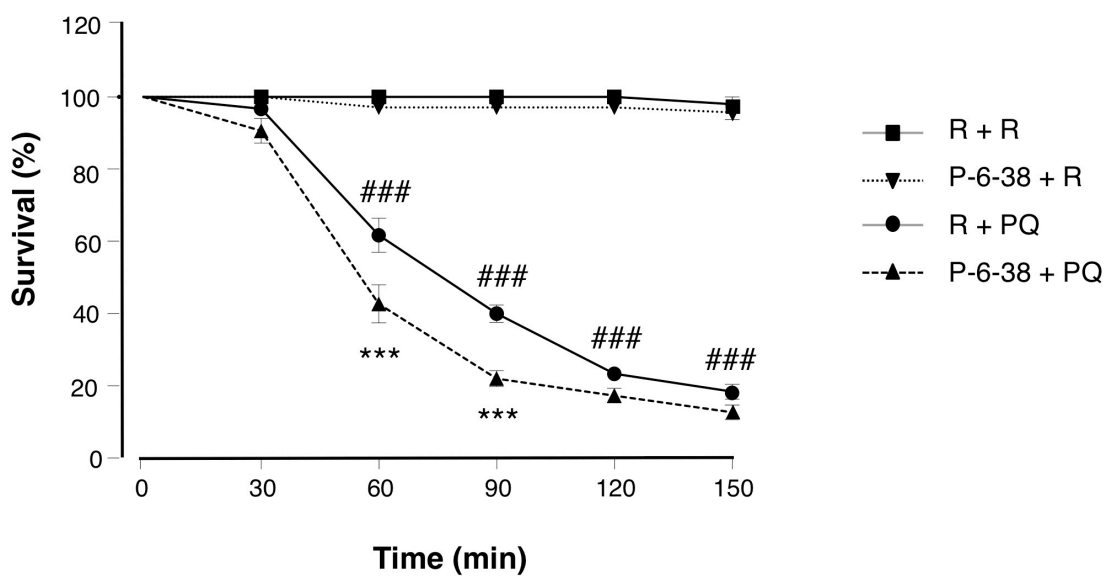

Hajji et al. Figure S2 


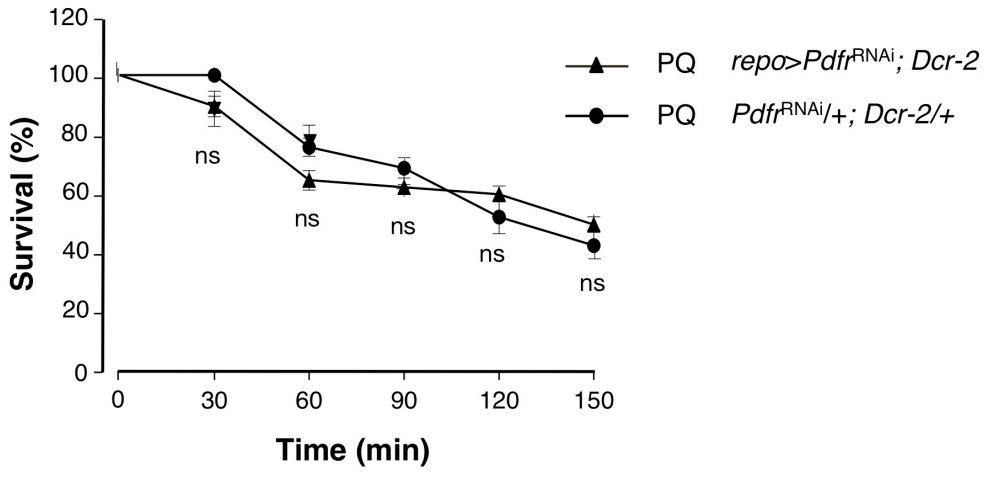

Hajji et al. Figure S3 

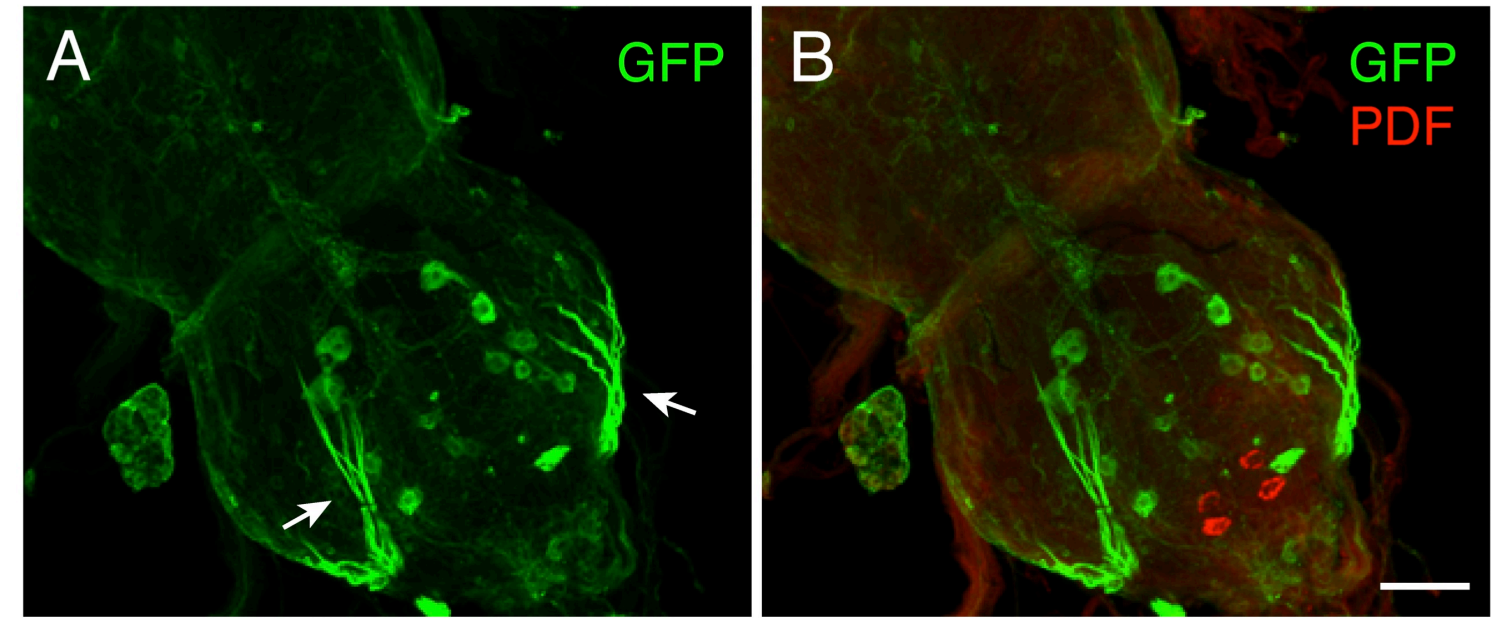

Hajii et al. Figure S4 


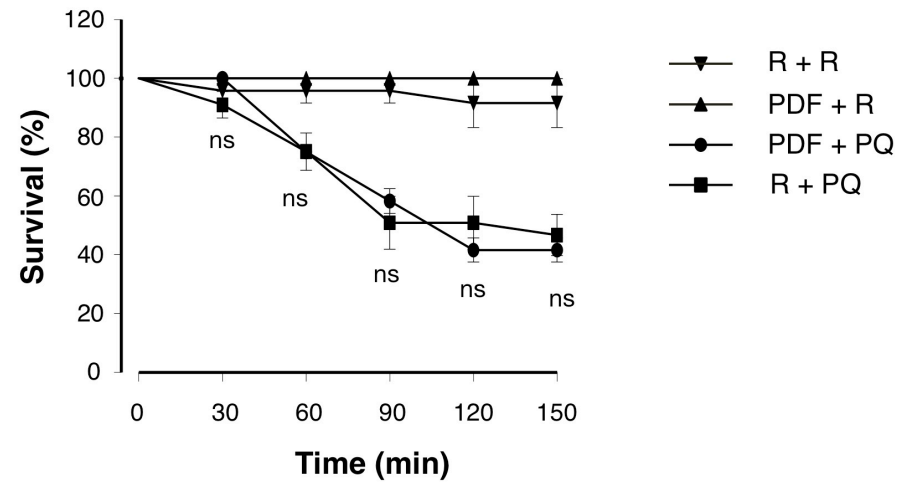

Hajji et al. Figure S5 